\title{
Integration of Physical Activity in Reablement for Community Dwelling Older Adults: A Systematic Scoping Review
}

This article was published in the following Dove Press journal: Journal of Multidisciplinary Healthcare

\author{
Hanne Leirbekk Mjøsund (iD) \\ Cathrine Fredriksen Moe iD ${ }^{1,2}$ \\ Elissa Burton (iD ${ }^{3}$ \\ Lisbeth Uhrenfeldt (iD) ${ }^{1,4}$ \\ 'Faculty of Nursing and Health Sciences, \\ Nord University, Bodø, Norway; ${ }^{2}$ Centre \\ for Work and Mental Health, Nordland \\ Hospital Trust, Bodø, Norway; ${ }^{3}$ School of \\ Physiotherapy \& Exercise Science, Curtin \\ University, Perth, Australia; ${ }^{4}$ Danish \\ Centre of Systematic Review, a JBI \\ Centre of Excellence, Center of Clinical \\ Guidelines, Department of Clinical \\ Medicine, Aalborg University, Aalborg, \\ Denmark
}

Background: Reablement is a rehabilitative intervention provided to homecare receivers with the aim of improving function and independence. There is limited evidence of the effectiveness of reablement, and the content of these interventions is variable. Physical activity (PA) is known to be important for improving and maintaining function among older adults, but it is unclear how PA is integrated in reablement.

Purpose: To map existing evidence of how PA strategies are integrated and explored in studies of reablement for community dwelling older adults and to identify knowledge gaps. Methods: An a priori protocol was published. Studies of time-limited, interdisciplinary reablement for community-dwelling older adults were considered for inclusion. Eight databases were searched for studies published between 1996 and June 2020, in addition to reference and citation searches. Study selection and data extraction were made independently by two reviewers.

Results: Fifty-one studies were included. Exercise strategies and practice of daily activities were included in the majority of intervention studies, but, in most cases, little information was provided about the intensity of PA. Interventions aiming to increase general PA levels or reduce sedentary behavior were rarely described. None of the studies explored older adults', healthcare providers' or family members' experiences with PA in a reablement setting, but some of the studies touched upon themes related to PA experiences. Some studies reported outcomes of physical fitness, including mobility, strength, and balance, but there was insufficient evidence for any synthesis of these results. None of the studies reported PA levels among older adults receiving reablement.

Conclusion: There is limited evidence of how PA is integrated and targeted to older adults' individual needs and preferences in a reablement setting. The feasibility and effectiveness of PA interventions, as well as experiences or barriers related to PA in a reablement setting, should be further investigated.

Keywords: rehabilitation, exercise therapy, health services for the aged, aged, 80 and over, physical fitness, health personnel

\section{Introduction}

Reablement is an interdisciplinary practice introduced in homecare services during the last two decades. The aim of reablement is to provide homecare services that help people (mostly older adults $>65$ years) regain function and independence, rather than continuing to increase services to compensate for ongoing functional loss. ${ }^{1-4}$ Different terms have been used for reablement such as everyday rehabilitation and restorative care. For the purpose of this study, the term reablement will be used and is
Correspondence: Hanne Leirbekk Mjøsund

Nord University, Universitetsallèen II,

Bodø 8026, Norway

Tel +4745968697

Email hanne.I.mjosund@nord.no
Journal of Multidisciplinary Healthcare 2020:13 I29|-13/5 
defined as rehabilitative initiatives that aim to maximize functional ability and independence among homecare service users, by offering intensive, time-limited, interdisciplinary, person-centered, and goal-directed homecare services. ${ }^{4}$ A common feature of reablement is that the services are person-centered, with an emphasis on identifying and working towards the participants' own prioritized goals. ${ }^{4}$ Different types of healthcare personnel (HCPs) are typically involved in reablement, including healthcare professionals (with a bachelor degree) such as occupational therapists (OTs), physical therapists (PTs), registered nurses (RNs), and also allied healthcare personnel (without a bachelor degree), eg, nurse assistants, students, or community healthcare workers. ${ }^{5}$

Reablement seems to meet political healthcare priorities by introducing innovative and sustainable initiatives that may improve functional ability and well-being in older age. ${ }^{6}$ However, the evidence-base supporting reablement is still limited. Several recent systematic reviews have investigated different perspectives of the effectiveness of reablement. ${ }^{1-5,7}$ Although some promising implications are reported, ${ }^{2,7}$ there is limited evidence of the effect of reablement for improving function and independence compared to standard home care. ${ }^{13-5}$ The specific characteristics of reablement provided in the included intervention studies are reported to be poorly described, and little is known about the effect of individual components included in reablement practice. ${ }^{3,4,7}$ The focus of this review was to map evidence on how physical activity (PA) is integrated in reablement, as PA is considered an important factor for improving and maintaining older adults' physical function. ${ }^{8}$

For the purpose of this study, physical activity was defined in accordance with the definition used by WHO as "any bodily movement produced by skeletal muscles that requires energy expenditure". 9 This includes different activities, such as leisure time PA, transportation, occupational activity, household activity, games, sports, everyday activities, and exercise. Within this broad definition of PA, the focus of this study was on general PA facilitation and also exercise strategies and prevention of sedentary behavior. Exercise was defined as

physical activity that is planned, structured, repetitive, and purposive in the sense that improvement or maintenance of one or more components of physical fitness is an objective. $^{10}$
Sedentary behavior was defined as "any waking behavior characterized by low energy expenditure [...] while in a sitting, reclining, or lying posture". ${ }^{11}$

Inactivity among older adults affects their physical fitness (eg, cardiorespiratory and muscular endurance, muscular strength, flexibility, balance, mobility, or speed of movement), and is correlated to frailty and functional limitations. ${ }^{12}$ Research demonstrates the positive effects of regular PA and exercise for older adults such as reduced risk of falling, ${ }^{13}$ reduced level of frailty, ${ }^{14}$ and improved performance in ADLs. ${ }^{15}$ WHO recommend older adults be physically active for at least 150 minutes a week, including activities that are adapted to the individuals' functional level. ${ }^{8}$ They also recommend activities that enhance muscle-strength and balance be included at least twice a week.

However, there are many factors that are perceived by older adults as barriers to being physically active. ${ }^{16,17}$ It is recommended that HCPs pay special attention to inform older adults about the health benefits of PA and that they consider the persons' personal, social, and environmental constraints for being physically active. ${ }^{16,18}$ Furthermore, it has been suggested that more attention be directed to reallife contexts of PA interventions among communitydwelling older adults. ${ }^{19}$

PA or exercise strategies are reported as part of the reablement intervention in some systematic reviews of reablement published over the last few years, ${ }^{2,4,5,7}$ but no further characteristics of these strategies are provided. No identified systematic review mentions strategies aimed at reducing sedentary behavior among participants. ${ }^{1-5,7}$ It was anticipated that experimental, quantitative, and qualitative study designs would provide evidence relevant to the objective of this scoping review and the intention was, therefore, to include a broader range of study designs than previously included in systematic reviews of reablement. A scoping review was considered appropriate in order to identify and map different types of evidence related to PA in reablement.

The objective of this systematic scoping review was to identify and map existing evidence of how PA strategies are integrated and explored in studies of reablement for community dwelling older adults and also to identify knowledge gaps that are important for further research.

More specific, the questions of this review were:

- To what extent have PA strategies been used in reablement for older adults and what are the reported characteristics of these strategies? 
- What evidence regarding experiences (of older adults, HCPs and family members) and barriers related to PA facilitation in a reablement setting can be identified?

- What is the scope of assessment methods used in relation to reablement that can inform about older adults' (changes in) PA behavior and physical fitness?

\section{Methods}

We used a systematic scoping review method following recommendations from the Joanna Briggs Institute. ${ }^{20,21}$ The study was reported based on the Preferred Reporting Items for Systematic reviews and Meta-Analyses extension for Scoping Reviews (PRISMA-ScR). ${ }^{22}$ A protocol was published prior to the review. ${ }^{23}$

\section{Eligibility Criteria Types of Participants}

To be included, the studies had to focus on older adults aged 65 years and over that were in receipt of reablement services. Studies focusing on HCPs working with reablement and family members of reablement participants were included. Studies focusing on people requiring end-of-life care were excluded.

\section{Concept}

Studies that investigated or explored the concept of reablement were included. The reablement intervention had to be delivered by several types of HCPs (involving at least two disciplines of healthcare professionals or one discipline in addition to allied healthcare personnel), aiming to improve functional ability and be person-centered (ie, targeted to the participants' individual goals). Studies investigating reablement with no time-restriction or a duration of more than 6 months were excluded. Studies focusing on home rehabilitation targeting people with one particular diagnosis (eg, heart failure, hip fractures, stroke, osteoarthritis) were also excluded. These particular criteria were not specified in the protocol, but were considered necessary in order to separate similar interventions that would not be considered reablement due to their specialized diagnostic focus. The criteria were decided upon prior to the selection process and used consistently throughout the selection. Studies were included regardless of whether or not they reported any information related to PA, since it was an aim to map both the existence of, and the absence of information related to PA in reablement studies.

\section{Context}

To be included, the reablement intervention had to be provided by homecare services (eg, managed by local government or not-for-profit agencies) in the participants' home (including a variety of housing arrangements) or local environment. Studies focusing on reablement interventions provided in long-term care facilities/nursing homes or housing arrangements with 24-hour care were excluded. Studies investigating reablement in relation to transition from a hospital setting were included if they met all other eligibility criteria. There were no restrictions regarding country of origin of the studies.

\section{Types of Sources}

This review included original peer-reviewed scientific studies with different designs, including (but not limited to) intervention studies (eg, Randomized Controlled Trials (RCTs), controlled trials, case control studies), qualitative studies, quantitative research, and mixed method research. Reviews, cost-effectiveness studies, and study protocols were excluded. Text (eg, political documents or government recommendations) and opinion papers were also excluded. Studies published in English, Norwegian, Danish, Swedish, and German were considered for inclusion. Reablement is a relatively young intervention with the majority of studies being published in the 2000s. ${ }^{1,2,4,5,24}$ Given the search by Cochrane et $\mathrm{al}^{4}$ had no date restrictions yet found few studies (ie, only those in the 2000s), we decided, like Ryburn et al, ${ }^{24}$ to only include studies published in 1996 or later.

\section{Search Strategy}

Firstly, an initial limited search in PubMed and CINAHL was undertaken to identify relevant key words and search terms used in titles and abstracts in studies published within the field. Secondly, based on search terms identified in this initial search, a search strategy was developed with assistance from a librarian. PubMed, Cochrane central register of controlled trials, Embase, PsycINFO, AMED, PEDro, CINAHL, and Google Scholar were all searched from 1996 to June 2020, with the latest update of the search being made on June 19, 2020. Although we initially intended to search for grey literature, we decided to only include peer-reviewed published studies, since there are no specific recommendations for inclusion of grey literature 
in scoping reviews. ${ }^{20}$ Therefore, we did not find it necessary to search ProQuest as reported in the protocol. The search strategy for PubMed is presented in Supplementary file 1 and includes search terms related to participants (aged/older adults) and concept (reablement). We did not include search terms related to context, to avoid narrowing the search and risk missing studies that did not explicitly describe the context. Relevant MeSH terms and headings were identified and used where required. Only English search terms were used in the search strategy. The language changed slightly depending on the database, however the main key words were used throughout. Thirdly, the reference lists of included studies were searched and a citation search of included studies was performed through Google Scholar in order to identify eligible studies that had not been identified through the previous search strategy.

\section{Study Selection}

After removing duplicates, two reviewers (HLM, CFM) independently performed screening of titles and abstracts and excluded studies not meeting the inclusion criteria. The remaining studies were retrieved in full-text and further evaluated for eligibility independently by two reviewers (HLM, CFM). Disagreements were solved by discussion or by involving a third reviewer for consensus (LU or EB). The authors of 24 studies were contacted for additional information related to our inclusion criteria (this information is specified in the result Tables). Rayyan ${ }^{25}$ and End Note X8 (Thomson Reuters, 2017) were used to manage records and data throughout the selection process.

\section{Data Charting Process}

Data charting forms were developed by the whole research team (HLM, CFM, EB, and LU) and piloted by two reviewers (HLM and CFM) prior to data extraction. Data extraction was made independently by HLM and CFM and then compared in order to reduce errors.

Extracted data included information about author, year, country, aim, study design/method, intervention and comparative intervention (if applicable), duration of intervention, HCPs involved, characteristics of reablement receivers, including age distribution if provided, sample size/informants. The following specific information related to the scoping review questions was extracted: Characteristics of general PA facilitation, exercise characteristics, experiences related to PA (by older adults, HCPs, or family members), and information of assessment of physical fitness and assessment of PA levels. An additional data extraction was made by HLM to identify PA-related terms used in each study.

Data mapping and summarization was conducted by one reviewer (HLM) in cooperation with the rest of the review team. For the second scoping question (experiences and barriers related to PA), meaningful units of text from the studies were extracted, condensed and systematized in an additional Excel spreadsheet. The findings were mapped and summarized and presented in text and tables.

\section{Results}

After screening 2527 unique records, of which 248 were examined in full-text, 51 articles met our eligibility criteria and served as the overall data material for the scoping review (illustrated in Figure 1, Prisma Flow Diagram). Among these studies were 15 intervention studies, including seven RCTs, ${ }^{26-32}$ five non-randomized controlled trials, ${ }^{33-37}$ and three non-controlled pre-post studies, ${ }^{38-40}$ in addition to one RCT long-term follow-up study; ${ }^{41}$ four studies with mixed design/other; ${ }^{42-45}$ four studies based on quantitative research; ${ }^{46-49}$ and 27 qualitative studies, of which 18 focused on HCPs' perspectives, ${ }^{50-66}$ seven on older adults' perspectives ${ }^{67-73}$ and two on family members perspectives. ${ }^{74,75}$

In most of the included studies, the terms reablement or restorative care were used $(n=47)$. However, four of the studies that met our inclusion criteria did use other intervention terms including "homecare rehabilitation service specially trained in falls identification", 37 "supported discharge team", 28 "everyday rehabilitation,"63 and "rehabilitative eldercare/homecare". ${ }^{62}$ Different groups of HCPs were represented in the interdisciplinary teams involved in the studies, including OTs (49 studies), PTs (42 studies), RNs (14 studies), or nurses (28 studies), allied healthcare personnel (46 studies), and social educators/managers (seven studies). The duration of reablement interventions varied between studies from 6 weeks or less (24 studies), 7 weeks -3 months ( 25 studies) or more than 3 months (two studies).

All of the included studies served as the collective data material for investigating if and how PA was described and explored in reablement research. Intervention studies are presented in Table 1, and studies that provided additional information about PA characteristics or provided information about PA experiences or barriers are presented in Table 2. Further information and study details of all included studies is presented in Supplementary file 2. 


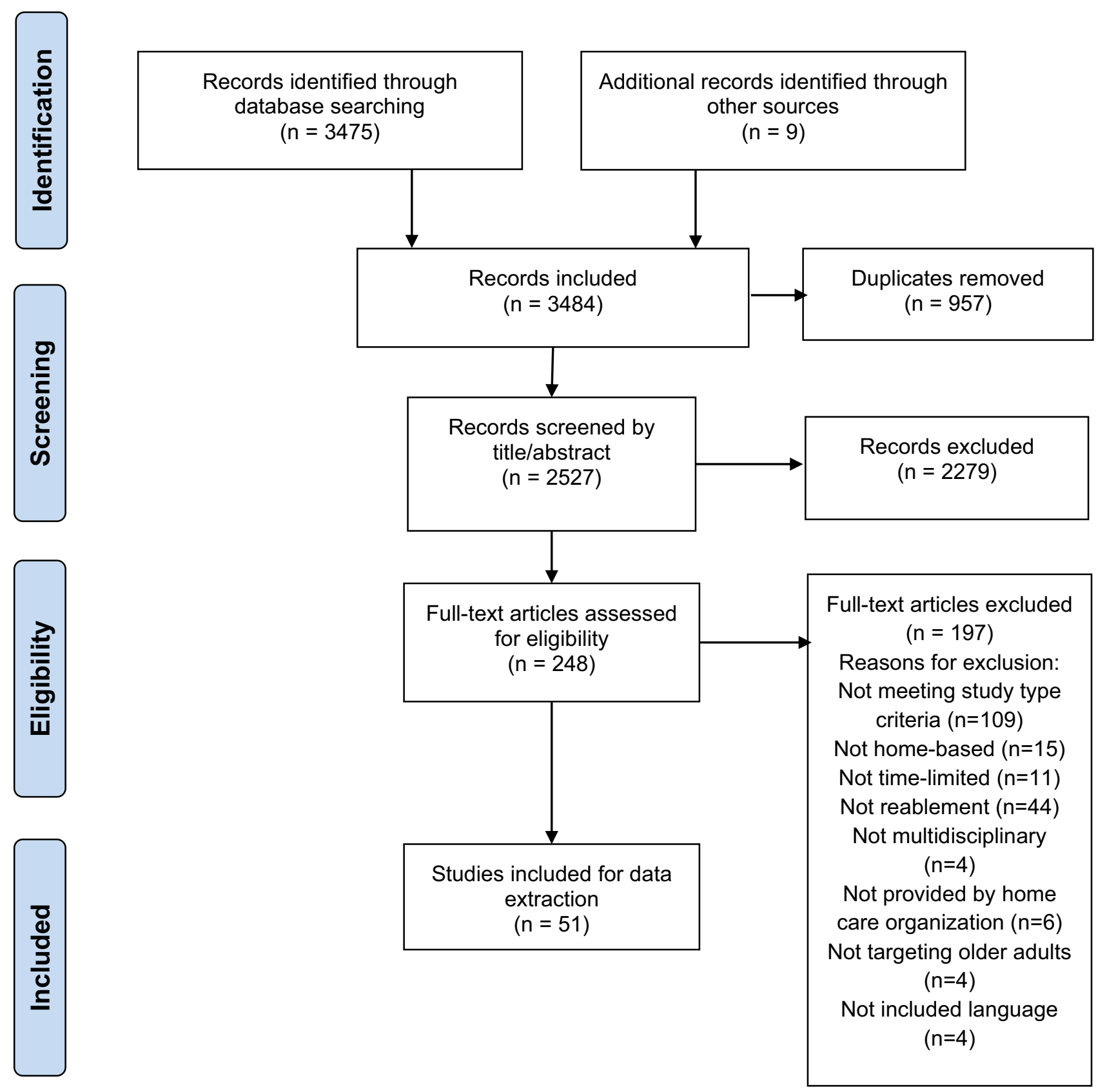

Figure I Prisma Flow diagram. (Adapted with permission from the PRISMA Group) Moher D, Liberati A, Tetzlaff J, Altman DG, The PRISMA Group (2009). Preferred Reporting Items for Systematic Reviews and Meta-Analyses: The PRISMA Statement. PLoS Med 6(7): e 1000097. doi:10.137I/journal.pmed I000097.The PRISMA Statement and the PRISMA Explanation and Elaboration document are distributed under the terms of the Creative Commons Attribution License, which permits unrestricted use, distribution, and reproduction in any medium, provided the original author and source are credited. (http://prisma-statement.org/PRISMAStatement/CitingAndUsingPRISMA.aspx). ${ }^{76}$

\section{QI: Extensiveness and Characteristics of Physical Activity Strategies in Reablement Characteristics of General PA Interventions}

Five Australian studies aimed to specifically investigate PArelated inquiries in a reablement setting. ${ }^{2641-43,46}$ In one of these studies, PA/Exercise interventions were described in detail, including intensity of the intervention, ${ }^{26}$ and two of the studies referred to government recommendations of PA in the background of the studies. ${ }^{42,43}$ Additionally, one Japanese RCT study reported including motivational interviews with the aim of assessing and encouraging physical activity (as one of several focuses of the interviews). ${ }^{31}$ With the exception of these studies, the term physical activity was rarely mentioned in other studies. Instead, a range of terms that were likely to involve some degree of PA were used, such as training, training in daily activities, practicing ADL- 


\begin{tabular}{|c|c|c|c|c|c|}
\hline 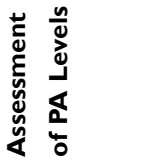 & & & 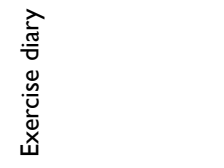 & z & z \\
\hline 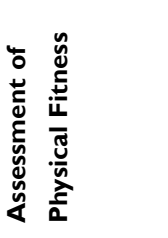 & & 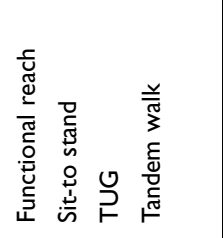 & 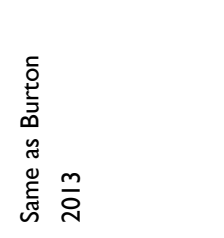 & 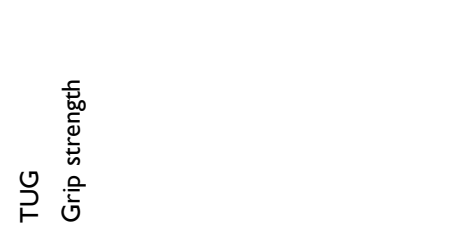 & 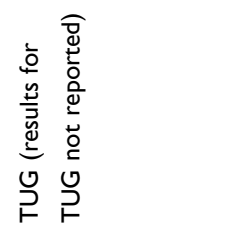 \\
\hline 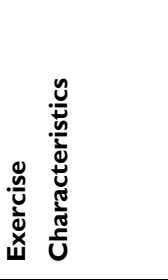 & & 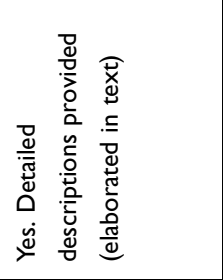 & 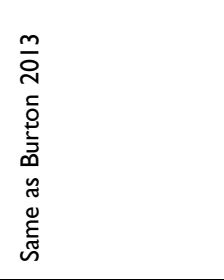 & 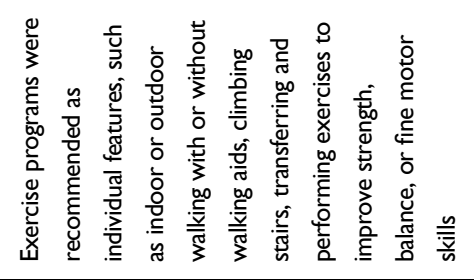 & 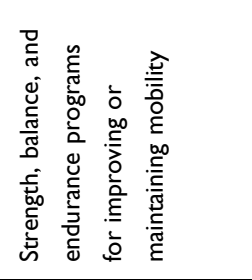 \\
\hline 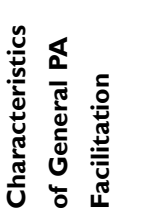 & & 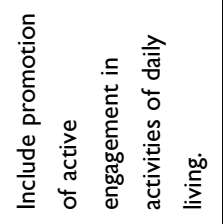 & 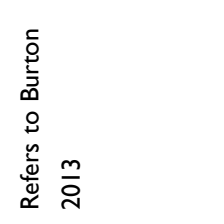 & 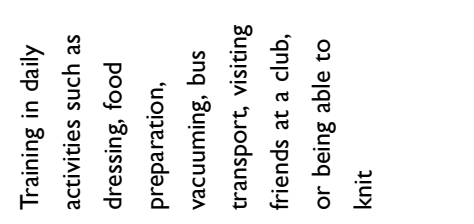 & 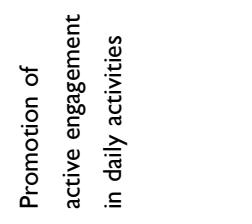 \\
\hline 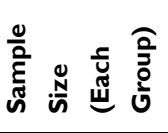 & & $\stackrel{\circ}{\stackrel{0}{q}} \widehat{o}$ & $\stackrel{\substack{f \\
\infty}}{a}$ & $\overline{\frac{\hat{m}}{m}}$ & 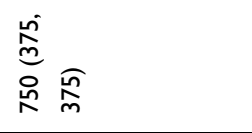 \\
\hline$\frac{n}{1}$ & & $\tilde{5}$ & 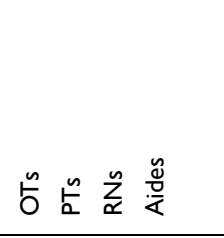 & 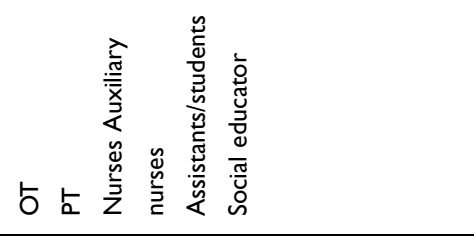 & 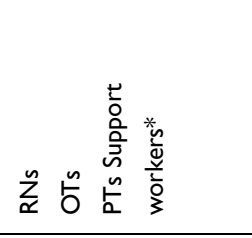 \\
\hline 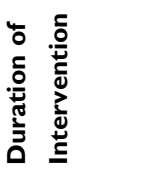 & & & 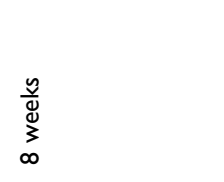 & 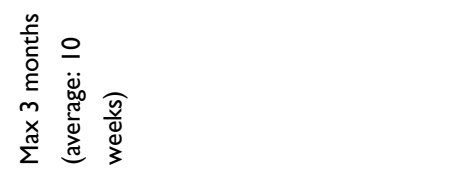 & 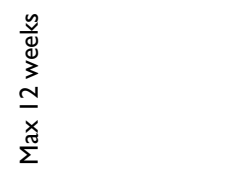 \\
\hline 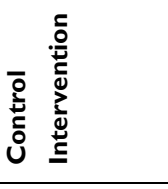 & & 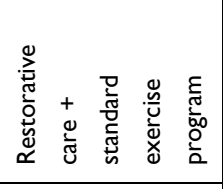 & 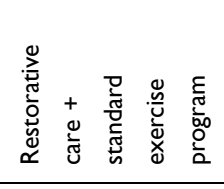 & 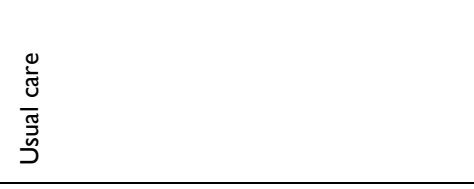 & 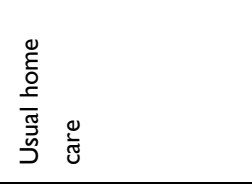 \\
\hline 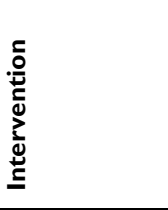 & & 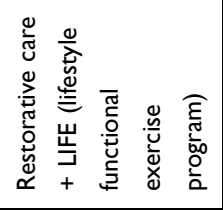 & 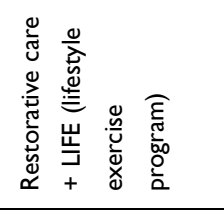 & 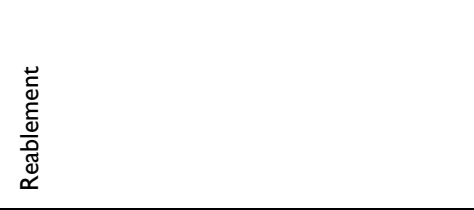 & 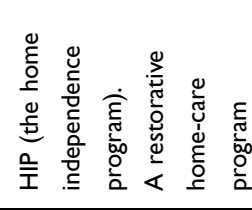 \\
\hline$\frac{.5}{\overline{0}}$ & 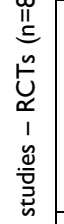 & 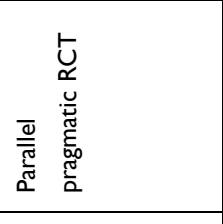 & 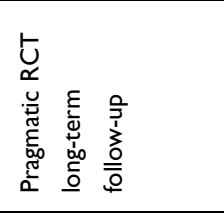 & 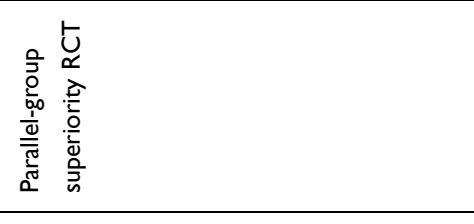 & $\underset{\propto}{\mathfrak{x}}$ \\
\hline 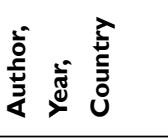 & 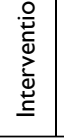 & 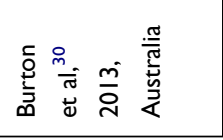 & 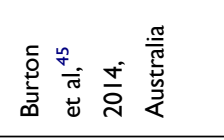 & 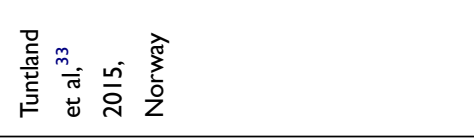 & 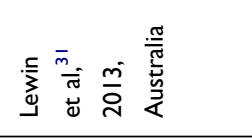 \\
\hline
\end{tabular}




\begin{tabular}{|c|c|c|c|c|c|}
\hline 운 & 우 & $\stackrel{0}{z}$ & 우 & & 우 \\
\hline$\stackrel{\circ}{z}$ & $\stackrel{0}{z}$ & $\stackrel{\circ}{z}$ & $\stackrel{0}{z}$ & & $\stackrel{৩}{\gtrless}$ \\
\hline 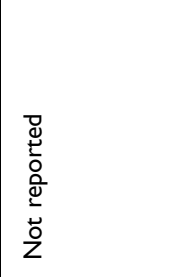 & 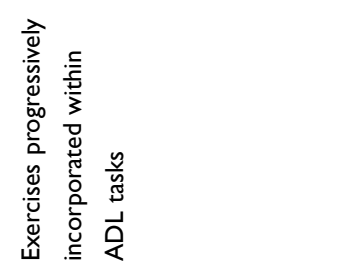 & 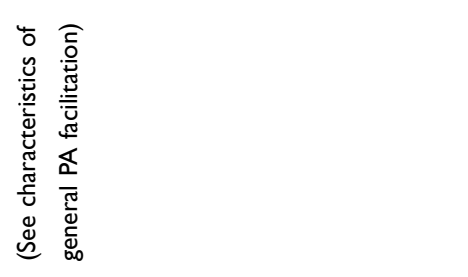 & 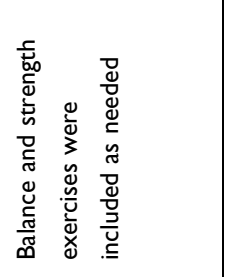 & & 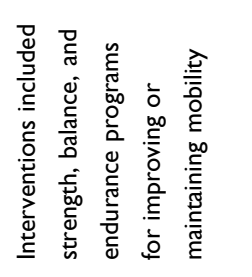 \\
\hline 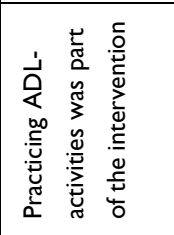 & 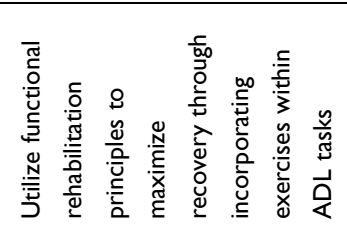 & 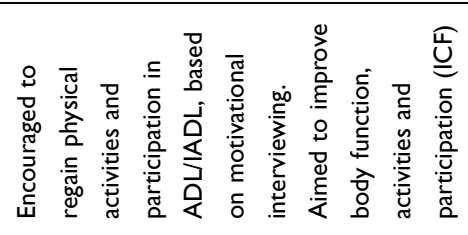 & 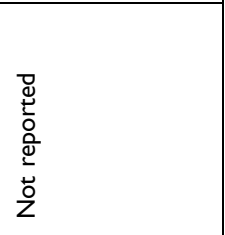 & & 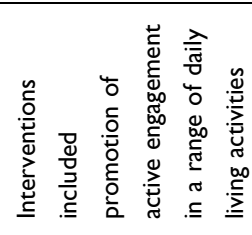 \\
\hline 일 & 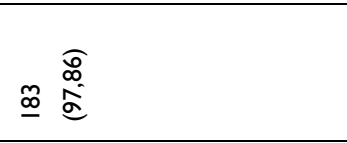 & 高 & $\begin{array}{l}\frac{\widehat{\sigma}}{\hat{\sigma}} \\
\underline{\underline{\alpha}}\end{array}$ & & 产 \\
\hline 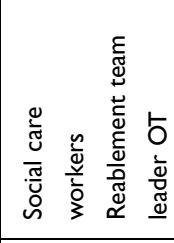 & 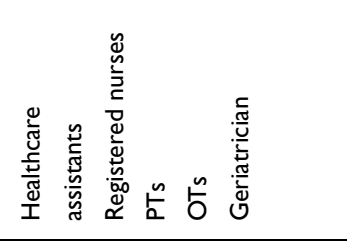 & 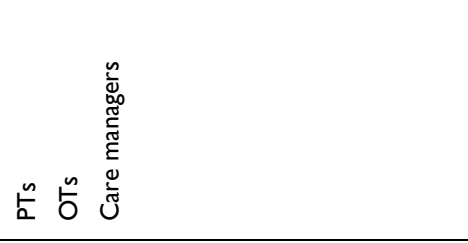 & 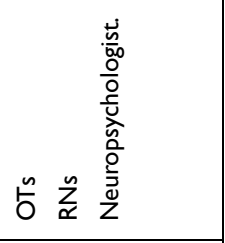 & & 总品 \\
\hline 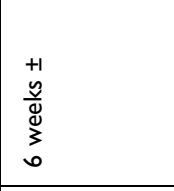 & 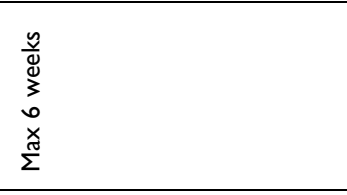 & 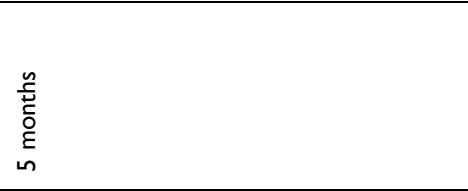 & 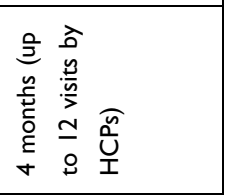 & & 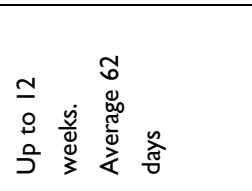 \\
\hline 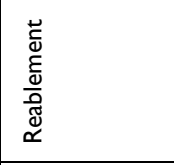 & 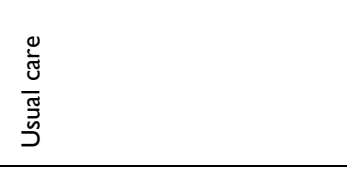 & 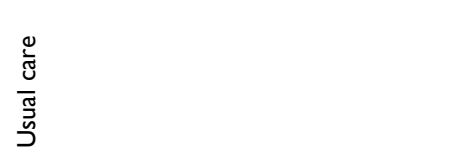 & 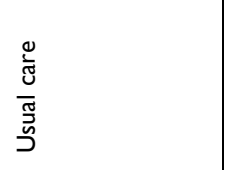 & & 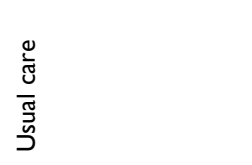 \\
\hline 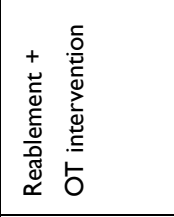 & 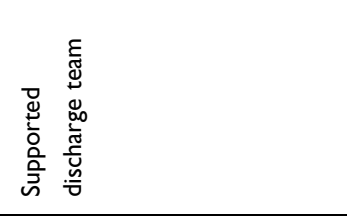 & 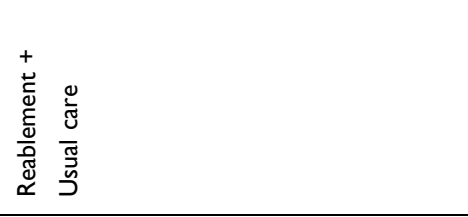 & 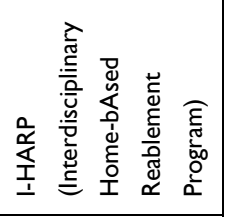 & 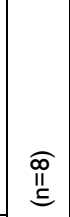 & 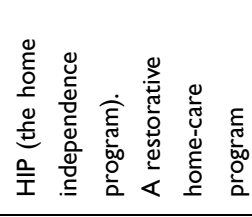 \\
\hline 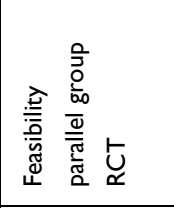 & $\underset{\longleftarrow}{t}$ & 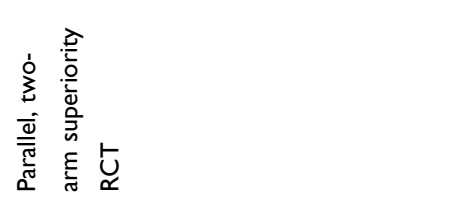 & 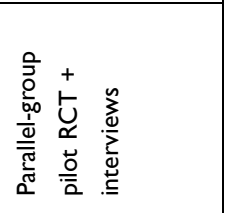 & 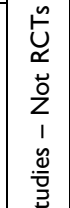 & 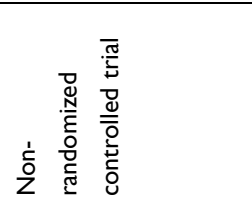 \\
\hline 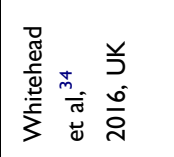 & 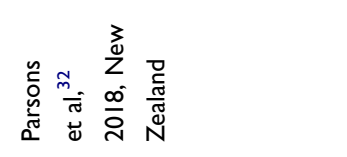 & 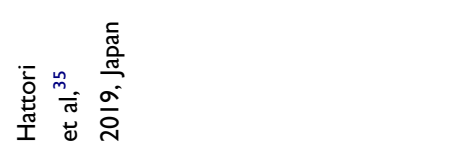 & 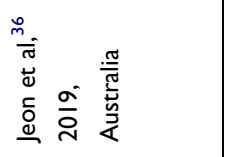 & 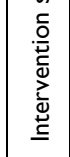 & 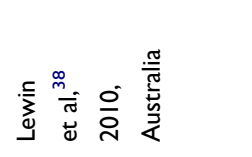 \\
\hline
\end{tabular}




\begin{tabular}{|c|c|c|c|}
\hline 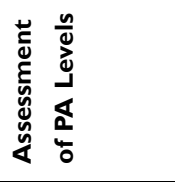 & $\stackrel{0}{z}$ & $\stackrel{0}{z}$ & $\stackrel{0}{z}$ \\
\hline 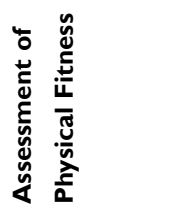 & $\stackrel{0}{z}$ & 우 & zo \\
\hline 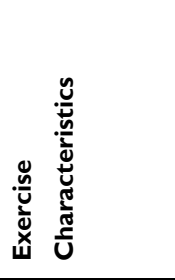 & 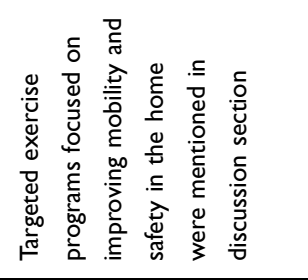 & 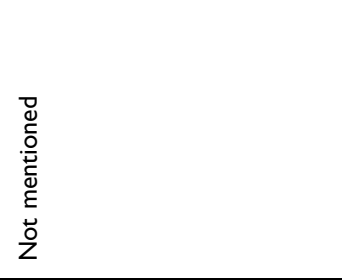 & 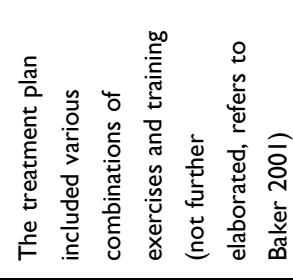 \\
\hline 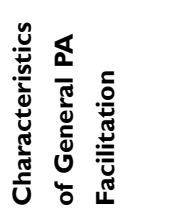 & 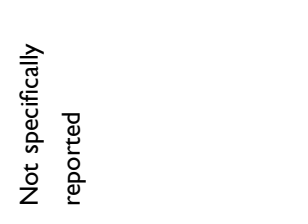 & 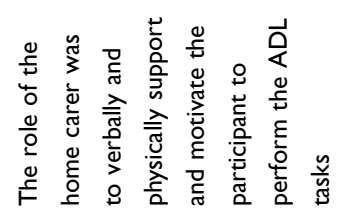 & 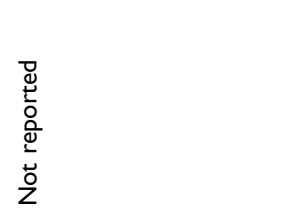 \\
\hline 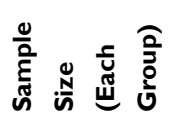 & 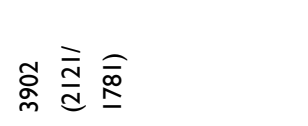 & $\bar{a}$ & 兽 \\
\hline$\frac{n}{0}$ & 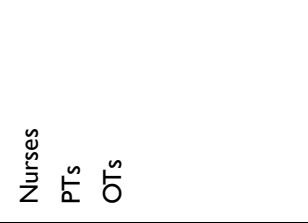 & 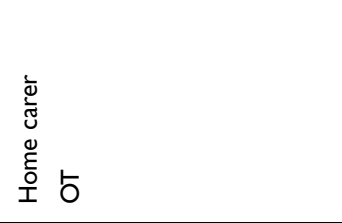 & 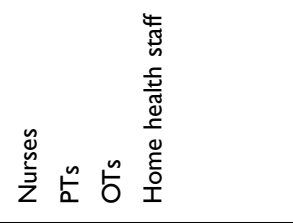 \\
\hline 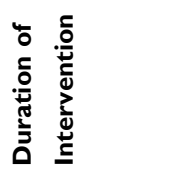 & 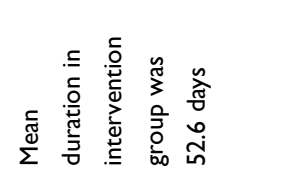 & 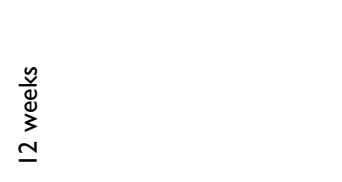 & 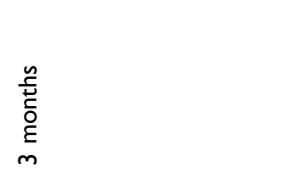 \\
\hline 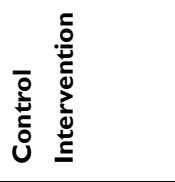 & 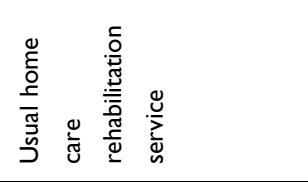 & 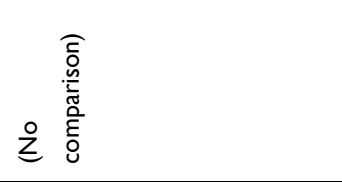 & 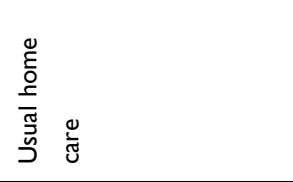 \\
\hline 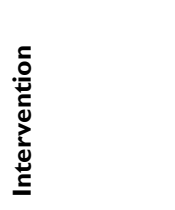 & 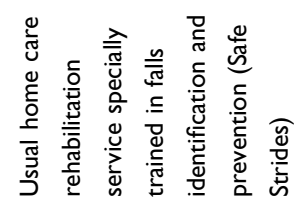 & 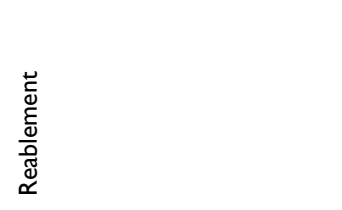 & 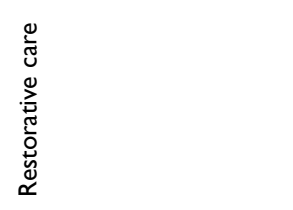 \\
\hline 高 & 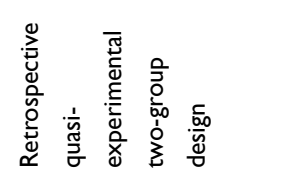 & 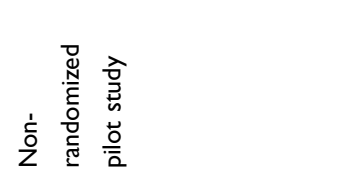 & 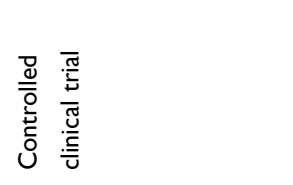 \\
\hline 竞 & 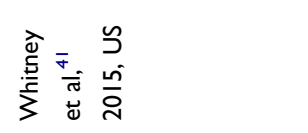 & 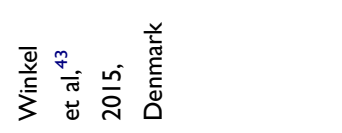 & 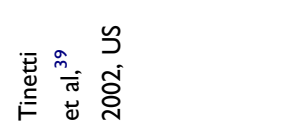 \\
\hline
\end{tabular}




\begin{tabular}{|c|c|c|c|}
\hline$\stackrel{\circ}{z}$ & $\stackrel{0}{z}$ & $\stackrel{\circ}{z}$ & $\stackrel{\circ}{z}$ \\
\hline 우 & $\frac{\infty}{\hat{a}}$ & $\stackrel{0}{z}$ & 우 \\
\hline 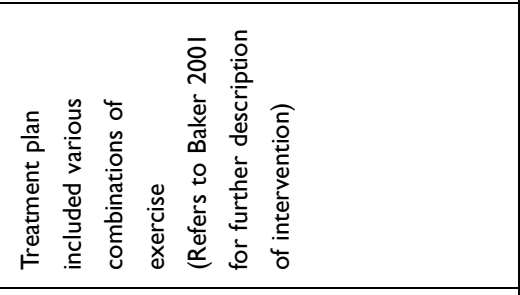 & 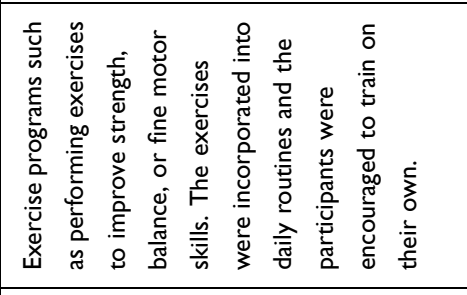 & 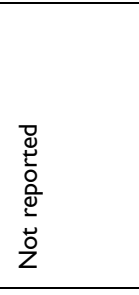 & 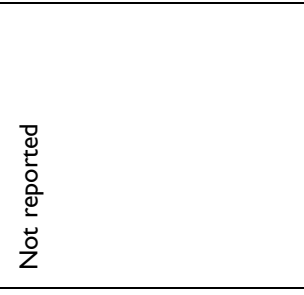 \\
\hline 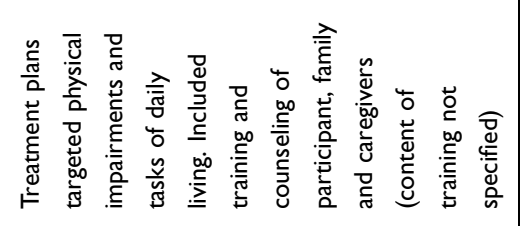 & 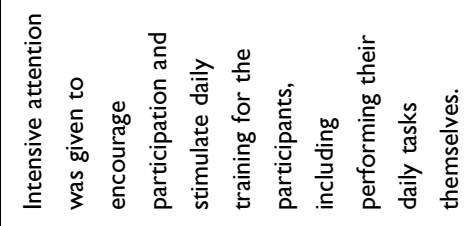 & 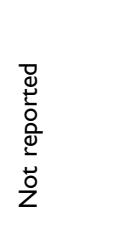 & 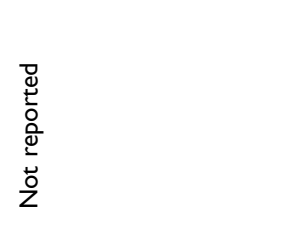 \\
\hline 高 & 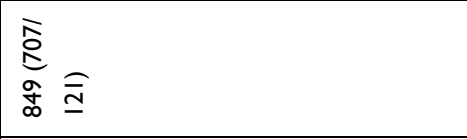 & $\stackrel{\circ}{7}$ & $\stackrel{\stackrel{\circ}{ }}{ }$ \\
\hline 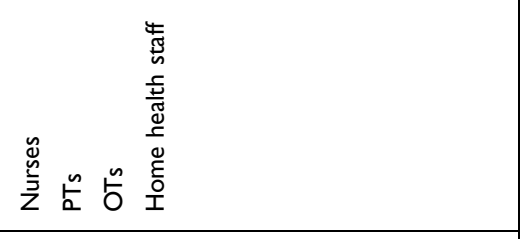 & 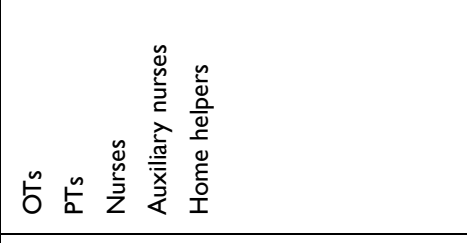 & 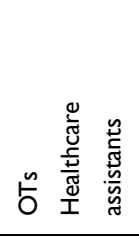 & 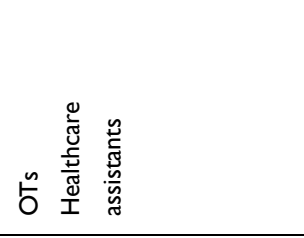 \\
\hline 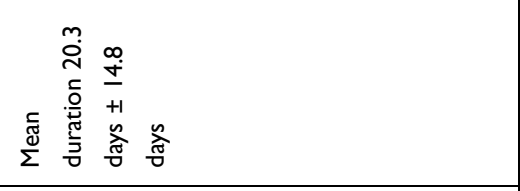 & 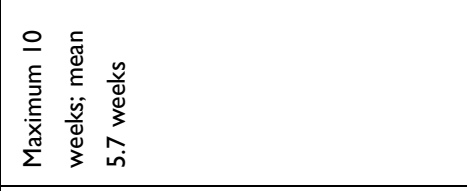 & 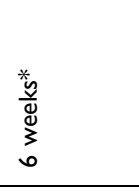 & 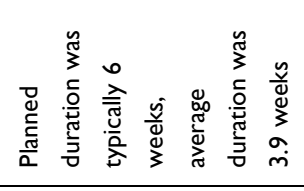 \\
\hline 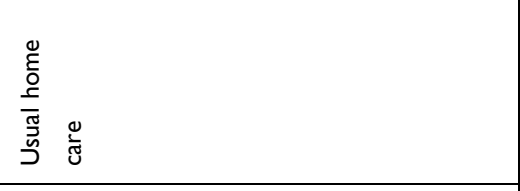 & 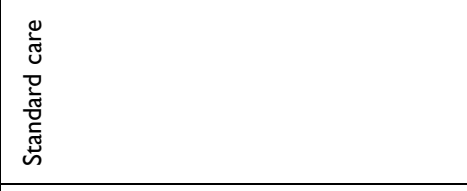 & 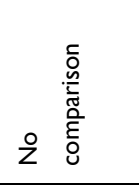 & 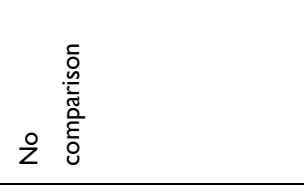 \\
\hline 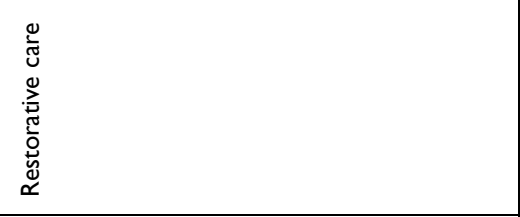 & 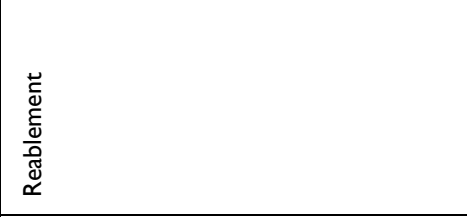 & 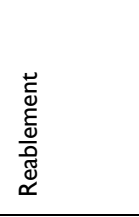 & 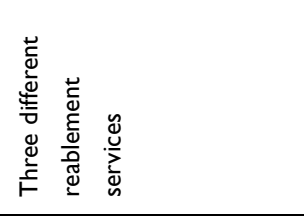 \\
\hline 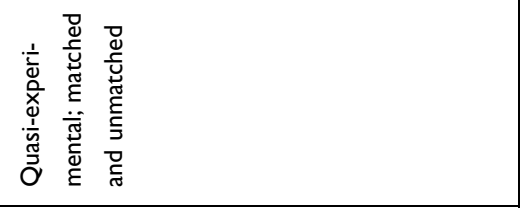 & 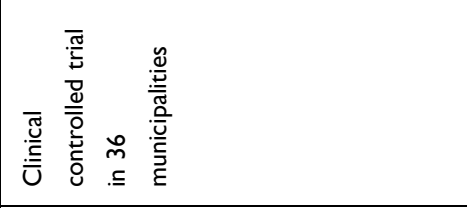 & 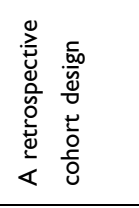 & 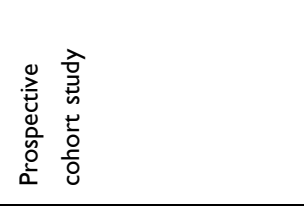 \\
\hline 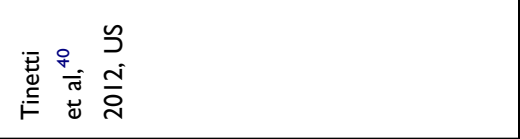 & 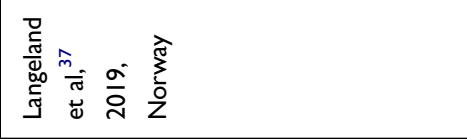 & 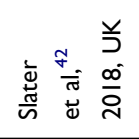 & 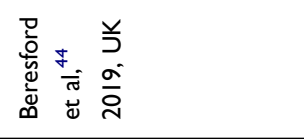 \\
\hline
\end{tabular}


Table 2 Other Studies Providing Information of PA Experiences and PA Characteristics

\begin{tabular}{|c|c|c|c|c|c|c|c|}
\hline Author & Aim & Method & $\begin{array}{l}\text { Duration of } \\
\text { Intervention }\end{array}$ & $\begin{array}{l}\text { HCPs } \\
\text { Involved }\end{array}$ & Informants & $\begin{array}{l}\text { PA } \\
\text { Characteristics }\end{array}$ & PA Experiences \\
\hline \multicolumn{8}{|c|}{ Perspectives of older adults } \\
\hline $\begin{array}{l}\text { Burton et al, }{ }^{46} \\
2013 \text {, Australia }\end{array}$ & $\begin{array}{l}\text { To identify the } \\
\text { motivators and } \\
\text { barriers to being } \\
\text { physically active for } \\
\text { older people } \\
\text { receiving either } \\
\text { restorative or } \\
\text { "usual" home care } \\
\text { services }\end{array}$ & $\begin{array}{l}\text { Cross-sectional } \\
\text { mixed method } \\
\text { study using } \\
\text { questionnaire } \\
\text { and interviews }\end{array}$ & $\begin{array}{l}\text { Minimum } 4 \\
\text { weeks, } \\
\text { generally } \\
6-12 \text { weeks* }\end{array}$ & $\begin{array}{l}\text { RNs } \\
\text { OTs } \\
\text { PTs Aides* }\end{array}$ & $\begin{array}{l}\text { Questionnaire: } 506 \\
\text { Older adults } \\
\text { Interviews: } 20 \\
\text { older adults }\end{array}$ & Not reported & $\begin{array}{l}\text { Facilitators/barriers } \\
\text { for PA among } \\
\text { previous reablement } \\
\text { receivers are } \\
\text { reported }\end{array}$ \\
\hline $\begin{array}{l}\text { Hjelle et al, } \\
2017, \text { Norway }\end{array}$ & $\begin{array}{l}\text { To describe older } \\
\text { adults experience } \\
\text { of reablement }\end{array}$ & $\begin{array}{l}\text { Qualitative } \\
\text { Interviews (part } \\
\text { of larger } \\
\text { research } \\
\text { program; } \\
\text { Tuntland 2015) }\end{array}$ & Max 3 months & $\begin{array}{l}\text { OT } \\
\text { PT } \\
\text { Nurses } \\
\text { Auxiliary } \\
\text { nurses } \\
\text { Assistants/ } \\
\text { students } \\
\text { Social } \\
\text { educator }\end{array}$ & 8 older adults & $\begin{array}{l}\text { Same as Tuntland } \\
2015\end{array}$ & $\begin{array}{l}\text { Own will-power and } \\
\text { responsibility is } \\
\text { considered important. } \\
\text { Encouragement and } \\
\text { motivation from HCPs } \\
\text { Home environment } \\
\text { stimulated to activity } \\
\text { Understanding of PA- } \\
\text { related terms }\end{array}$ \\
\hline $\begin{array}{l}\text { Wilde et al, }{ }^{77} \\
2012 \text {, UK }\end{array}$ & $\begin{array}{l}\text { Not clearly stated, } \\
\text { but the title informs } \\
\text { that the } \\
\text { perceptions and } \\
\text { experience of users } \\
\text { of home-care } \\
\text { reablement services } \\
\text { are in focus }\end{array}$ & Interviews & $\begin{array}{l}\text { Normally up } \\
\text { to } 6 \text { weeks, } \\
\text { with some } \\
\text { flexibility* }\end{array}$ & $\begin{array}{l}\text { Homecare } \\
\text { staff with } \\
\text { additional } \\
\text { training. } \\
\text { OTs regarded } \\
\text { as essential } \\
\text { members of } \\
\text { the team* }\end{array}$ & $\begin{array}{l}34 \text { service users } \\
10 \text { carers }\end{array}$ & $\begin{array}{l}\text { Focuses on } \\
\text { increasing service } \\
\text { users ability to } \\
\text { perform tasks such } \\
\text { as getting up, } \\
\text { washing, bathing, } \\
\text { moving around the } \\
\text { home and other daily } \\
\text { living activities such } \\
\text { as preparing drinks } \\
\text { and light meals }\end{array}$ & $\begin{array}{l}\text { Expressed frustration } \\
\text { at lack of professional } \\
\text { expertise to improve/ } \\
\text { maintain outdoor } \\
\text { mobility }\end{array}$ \\
\hline $\begin{array}{l}\text { Moe \& } \\
\text { Brinchmann, }{ }^{75} \\
\text { 2016, Norway }\end{array}$ & $\begin{array}{l}\text { To generate } \\
\text { a grounded theory } \\
\text { of service users' } \\
\text { and their } \\
\text { caregivers' } \\
\text { experiences of } \\
\text { reablement }\end{array}$ & $\begin{array}{l}\text { Grounded } \\
\text { theory; focus } \\
\text { groups, } \\
\text { interviews and } \\
\text { observation }\end{array}$ & $\begin{array}{l}\text { Average } \\
\text { duration of } 6 \\
\text { weeks, } \\
\text { maximal } \\
\text { duration of } 6 \\
\text { months* }\end{array}$ & $\begin{array}{l}\text { Nurse } \\
\text { OTs } \\
\text { PTs } \\
\text { Nurse } \\
\text { assistants }\end{array}$ & $\begin{array}{l}17 \text { services users, } \\
10 \text { carers }\end{array}$ & $\begin{array}{l}\text { Includes doing } \\
\text { repetitive practice } \\
\text { of activities of daily } \\
\text { life at home and in } \\
\text { the neighborhood. } \\
\text { Exercises included } \\
\text { based on a detailed } \\
\text { screening that } \\
\text { identifies activity } \\
\text { goals and functional } \\
\text { impairments, with } \\
\text { a focus on physical } \\
\text { strengthening }\end{array}$ & $\begin{array}{l}\text { Values/knowledge of } \\
\text { PA are considered. } \\
\text { Physical strengthening } \\
\text { could boost } \\
\text { participation in other } \\
\text { activities. } \\
\text { Experiences of } \\
\text { insecurity for injury } \\
\text { and overload. } \\
\text { Self-confidence for PA } \\
\text { was build during the } \\
\text { reablement. } \\
\text { Encouragement and } \\
\text { motivation from HCPs } \\
\text { was important. } \\
\text { Reducing } \\
\text { environmental barriers } \\
\text { (indoors/outdoors) }\end{array}$ \\
\hline
\end{tabular}

(Continued) 
Table 2 (Continued).

\begin{tabular}{|c|c|c|c|c|c|c|c|}
\hline Author & Aim & Method & $\begin{array}{l}\text { Duration of } \\
\text { Intervention }\end{array}$ & $\begin{array}{l}\text { HCPs } \\
\text { Involved }\end{array}$ & Informants & $\begin{array}{l}\text { PA } \\
\text { Characteristics }\end{array}$ & PA Experiences \\
\hline $\begin{array}{l}\text { Tuntland } \\
\text { et al, }{ }^{51} 2019 \text {, } \\
\text { Norway }\end{array}$ & $\begin{array}{l}\text { To explore which } \\
\text { occupations/ } \\
\text { activities older } \\
\text { people with } \\
\text { functional decline } \\
\text { find important to } \\
\text { improve, which of } \\
\text { these they } \\
\text { prioritize as their } \\
\text { rehabilitation goals, } \\
\text { and what factors } \\
\text { are associated with } \\
\text { these priorities }\end{array}$ & $\begin{array}{l}\text { A cross- } \\
\text { sectional study } \\
\text { based on data } \\
\text { from } \\
\text { a nationwide } \\
\text { trial (Langeland } \\
2019)\end{array}$ & $\begin{array}{l}\text { Mean } 5.7 \\
\text { weeks } \\
\text { (majority } \\
\text { between } 4 \\
\text { and } 6 \text { weeks) }\end{array}$ & $\begin{array}{l}\text { OTs } \\
\text { PTs } \\
\text { Nurses } \\
\text { Auxiliary } \\
\text { nurse } \\
\text { Home helpers }\end{array}$ & $\begin{array}{l}738 \text { reablement } \\
\text { receivers }\end{array}$ & $\begin{array}{l}\text { Same as Langeland } \\
2019\end{array}$ & $\begin{array}{l}\text { Goals related to } \\
\text { functional mobility } \\
\text { was most often } \\
\text { prioritized, followed } \\
\text { by goals related to } \\
\text { personal care and } \\
\text { household activities }\end{array}$ \\
\hline $\begin{array}{l}\text { Whitehead } \\
\text { et al, }{ }^{48} 2018 \text {, } \\
\text { UK }\end{array}$ & $\begin{array}{l}\text { To provide } \\
\text { a detailed } \\
\text { description of the } \\
\text { content of the } \\
\text { occupational } \\
\text { therapy } \\
\text { intervention that } \\
\text { was provided in the } \\
\text { OTHERS trial, and } \\
\text { to evaluate } \\
\text { whether the } \\
\text { intervention was } \\
\text { acceptable to the } \\
\text { participants who } \\
\text { received it }\end{array}$ & $\begin{array}{l}\text { Feasibility/ } \\
\text { evaluation study } \\
\text { (Part of } \\
\text { a feasibility } \\
\text { RCT); } \\
\text { questionnaire } \\
\text { and interviews }\end{array}$ & $\begin{array}{l}\text { Median length } \\
\text { of reablement } \\
\text { episode was } \\
56 \text { days } \\
\text { (range: } \\
20-126 \text { days) }\end{array}$ & $\begin{array}{l}\text { OT } \\
\text { Social care } \\
\text { reablement } \\
\text { workers }\end{array}$ & $\begin{array}{l}\text { Interviews: } 5 \text { older } \\
\text { adults } \\
\text { Questionnaire: } 8 \\
\text { older adults }\end{array}$ & $\begin{array}{l}\text { Practicing ADL } \\
\text { activities was } \\
\text { included in } \\
\text { intervention }\end{array}$ & $\begin{array}{l}\text { Outdoor mobility } \\
\text { goals were difficult to } \\
\text { reach due to } \\
\text { fluctuations of health } \\
\text { condition or weather }\end{array}$ \\
\hline $\begin{array}{l}\text { Magne et al, }{ }^{78} \\
2020, \text { Norway }\end{array}$ & $\begin{array}{l}\text { To describe how } \\
\text { older adults engage } \\
\text { in daily activities } \\
\text { within the context } \\
\text { of receiving } \\
\text { reablement and to } \\
\text { explore } \\
\text { participation in } \\
\text { daily activities }\end{array}$ & Interviews & $\begin{array}{l}6 \text { weeks or } \\
\text { less, with } \\
\text { some } \\
\text { exceptions* }\end{array}$ & $\begin{array}{l}\text { OTs } \\
\text { PTs } \\
\text { Homecare } \\
\text { workers }\end{array}$ & 10 Older adults. & Not reported & $\begin{array}{l}\text { Describes and } \\
\text { explores older adults' } \\
\text { experiences of } \\
\text { engaging in daily } \\
\text { activities and } \\
\text { exercises when } \\
\text { receiving reablement }\end{array}$ \\
\hline \multicolumn{8}{|c|}{ Perspectives of HCPs } \\
\hline $\begin{array}{l}\text { Hjelle et al, }{ }^{62} \\
2018, \text { Norway }\end{array}$ & $\begin{array}{l}\text { To explore and } \\
\text { describe the roles } \\
\text { of interdisciplinary } \\
\text { teams in } \\
\text { reablement services } \\
\text { in a Norwegian } \\
\text { setting }\end{array}$ & $\begin{array}{l}\text { Focus groups + } \\
\text { interviews }\end{array}$ & $\begin{array}{l}\text { Max } 3 \text { months } \\
\text { in the rural } \\
\text { setting, } 4 \\
\text { weeks } \\
\text { duration in } \\
\text { the city }\end{array}$ & $\begin{array}{l}\text { OTs } \\
\text { PTs } \\
\text { Social } \\
\text { educators } \\
\text { Nurses } \\
\text { Auxiliary } \\
\text { nurses } \\
\text { Assistants }\end{array}$ & $\begin{array}{l}27 \text { HCPs (PTs, } \\
\text { OTs, Nurses, } \\
\text { Auxiliary nurses) }\end{array}$ & $\begin{array}{l}\text { Same as Tuntland } \\
2015\end{array}$ & $\begin{array}{l}\text { HCPs considered the } \\
\text { reablement } \\
\text { organization to be } \\
\text { beneficial for reaching } \\
\text { more users }\end{array}$ \\
\hline
\end{tabular}

(Continued) 
Table 2 (Continued).

\begin{tabular}{|c|c|c|c|c|c|c|c|}
\hline Author & Aim & Method & $\begin{array}{l}\text { Duration of } \\
\text { Intervention }\end{array}$ & $\begin{array}{l}\text { HCPs } \\
\text { Involved }\end{array}$ & Informants & $\begin{array}{l}\text { PA } \\
\text { Characteristics }\end{array}$ & PA Experiences \\
\hline $\begin{array}{l}\text { Jakobsen } \\
\text { et al, }{ }^{64} 2018, \\
\text { Norway }\end{array}$ & $\begin{array}{l}\text { To describe HCPs } \\
\text { perspectives of } \\
\text { next of kin in the } \\
\text { context of } \\
\text { reablement }\end{array}$ & Focus groups & $\begin{array}{l}\text { Often 4-6 } \\
\text { weeks }\end{array}$ & $\begin{array}{l}\text { PTs } \\
\text { OTs } \\
\text { RNs } \\
\text { Social } \\
\text { educators } \\
\text { Other } \\
\text { employees of } \\
\text { the home care } \\
\text { services }\end{array}$ & $\begin{array}{l}49 \mathrm{HCPs} \\
\text { (RNs, Health } \\
\text { workers, OTs, } \\
\text { students, PTs, } \\
\text { Social educators) }\end{array}$ & $\begin{array}{l}\text { HCPs assisted the } \\
\text { older adults with } \\
\text { practicing everyday } \\
\text { activities and an } \\
\text { individual physical } \\
\text { training program }\end{array}$ & $\begin{array}{l}\text { Values/knowledge of } \\
\text { PA are considered. } \\
\text { Relatives as } \\
\text { a facilitator/barrier } \\
\text { for PA. }\end{array}$ \\
\hline $\begin{array}{l}\text { Meldgaard } \\
\text { Hansen, } \\
2016, \\
\text { Denmark }\end{array}$ & $\begin{array}{l}\text { To analyze and } \\
\text { discuss how the } \\
\text { bodywork of } \\
\text { homecare develops } \\
\text { and is framed as } \\
\text { clean, non-dirty } \\
\text { work in the context } \\
\text { of rehabilitative } \\
\text { homecare }\end{array}$ & $\begin{array}{l}\text { Ethnographic } \\
\text { fieldwork }\end{array}$ & $\begin{array}{l}\text { Average of } 8 \\
\text { weeks in one } \\
\text { unit, not } \\
\text { specified in } \\
\text { the other } \\
\text { unit* }\end{array}$ & $\begin{array}{l}\text { PTs } \\
\text { OTs } \\
\text { Nurses Social } \\
\text { and healthcare } \\
\text { workers }\end{array}$ & $\begin{array}{l}\text { Two homecare } \\
\text { units; } 30 \text { interviews } \\
\text { with homecare } \\
\text { workers, managers } \\
\text { and administrators, } \\
\text { shadow } \\
\text { observations of } 20 \\
\text { homecare workers }\end{array}$ & Not reported & $\begin{array}{l}\text { HCPs experienced } \\
\text { transformation of } \\
\text { roles }\end{array}$ \\
\hline $\begin{array}{l}\text { Eliassen et al, } \\
2018 \text {, Norway }\end{array}$ & $\begin{array}{l}\text { To explore how } \\
\text { physiotherapy } \\
\text { practice is } \\
\text { performed in } \\
\text { reablement settings } \\
\text { and the content of } \\
\text { the service } \\
\text { provided to } \\
\text { reablement users }\end{array}$ & Field study & $\begin{array}{l}\text { 4-6 weeks } \\
\text { (with some } \\
\text { exceptions)* }\end{array}$ & $\begin{array}{l}\text { PTs } \\
\text { OTs } \\
\text { Nurses } \\
\text { Allied health } \\
\text { personnel* }\end{array}$ & $\begin{array}{l}7 \text { PTs and } 7 \text { allied } \\
\text { health personnel } \\
\text { (+ } 7 \text { clients) }\end{array}$ & $\begin{array}{l}\text { Daily activities } \\
\text { were included in } \\
\text { reablement plans } \\
\text { and could involve } \\
\text { getting dressed, } \\
\text { showering, and } \\
\text { walking to the } \\
\text { grocery store. } \\
\text { Exercises were } \\
\text { provided in all } \\
\text { teams, either as } \\
\text { mainly standardized } \\
\text { exercises or } \\
\text { individual adapted } \\
\text { exercises. } \\
\text { The exercises } \\
\text { mainly targeted } \\
\text { balance, leg } \\
\text { strength, and gait } \\
\text { endurance }\end{array}$ & $\begin{array}{l}\text { Experienced } \\
\text { beneficial } \\
\text { organization for } \\
\text { reaching more users } \\
\text { Competencies of } \\
\text { allied health } \\
\text { personnel was } \\
\text { considered an } \\
\text { important factor. } \\
\text { Simple exercises } \\
\text { were considered } \\
\text { beneficial }\end{array}$ \\
\hline $\begin{array}{l}\text { Eliassen et al, }{ }^{57} \\
2018 \text {, Norway }\end{array}$ & $\begin{array}{l}\text { To explore how the } \\
\text { allied health } \\
\text { personnel follow- } \\
\text { up instructions and } \\
\text { supervision by PTs } \\
\text { in reablement }\end{array}$ & Field study & $\begin{array}{l}\text { 4-6 weeks } \\
\text { (with some } \\
\text { exceptions)* }\end{array}$ & $\begin{array}{l}\text { PTs } \\
\text { OTs } \\
\text { Nurses } \\
\text { Allied health } \\
\text { personnel* }\end{array}$ & $\begin{array}{l}7 \text { PTs and } 7 \text { allied } \\
\text { health personnel } \\
\text { (+ } 7 \text { clients) }\end{array}$ & $\begin{array}{l}\text { Same as Eliassen } \\
2018 a\end{array}$ & $\begin{array}{l}\text { Competencies of } \\
\text { allied health } \\
\text { personnel was } \\
\text { considered an } \\
\text { important factor. } \\
\text { Individually targeted } \\
\text { exercises were } \\
\text { preferred in some } \\
\text { teams }\end{array}$ \\
\hline
\end{tabular}

(Continued) 
Table 2 (Continued).

\begin{tabular}{|c|c|c|c|c|c|c|c|}
\hline Author & Aim & Method & $\begin{array}{l}\text { Duration of } \\
\text { Intervention }\end{array}$ & $\begin{array}{l}\text { HCPs } \\
\text { Involved }\end{array}$ & Informants & $\begin{array}{l}\text { PA } \\
\text { Characteristics }\end{array}$ & PA Experiences \\
\hline $\begin{array}{l}\text { Eliassen et al, }{ }^{59} \\
2018, \text { Norway }\end{array}$ & $\begin{array}{l}\text { To explore the } \\
\text { content of PTs' } \\
\text { supervision of } \\
\text { home trainers in } \\
\text { reablement teams }\end{array}$ & Field study & $\begin{array}{l}\text { 4-6 weeks } \\
\text { (with some } \\
\text { exceptions) * }\end{array}$ & $\begin{array}{l}\text { PTs } \\
\text { OTs } \\
\text { Nurses } \\
\text { Allied health } \\
\text { personnel* }\end{array}$ & $\begin{array}{l}7 \text { PTs and } 7 \text { allied } \\
\text { health personnel (+ } \\
7 \text { clients) }\end{array}$ & $\begin{array}{l}\text { Same as Eliassen } \\
2018 a\end{array}$ & $\begin{array}{l}\text { Competencies of } \\
\text { allied health } \\
\text { personnel was } \\
\text { considered an } \\
\text { important factor }\end{array}$ \\
\hline $\begin{array}{l}\text { Eliassen et al, }{ }^{70} \\
2020, \text { Norway }\end{array}$ & $\begin{array}{l}\text { To investigate and } \\
\text { discuss how PTs on } \\
\text { reablement teams } \\
\text { plan and adapt } \\
\text { training } \\
\text { interventions to } \\
\text { enhance users' } \\
\text { functional abilities }\end{array}$ & Field study & $\begin{array}{l}\text { 4-6 weeks } \\
\text { (with some } \\
\text { exceptions) * }\end{array}$ & $\begin{array}{l}\text { PTs } \\
\text { OTs } \\
\text { Nurses } \\
\text { Allied health } \\
\text { personne** }\end{array}$ & $\begin{array}{l}7 \text { PTs and } 7 \text { allied } \\
\text { health personnel (+ } \\
7 \text { clients) }\end{array}$ & $\begin{array}{l}\text { Identified three } \\
\text { main categories of } \\
\text { interventions: (i) } \\
\text { exercise-based } \\
\text { training, (ii) activity- } \\
\text { based training, or } \\
\text { (iii) a combination } \\
\text { of both exercise } \\
\text { and activity-based } \\
\text { training }\end{array}$ & Not reported \\
\hline $\begin{array}{l}\text { Bodker et al, } \\
2019, \\
\text { Denmark }\end{array}$ & $\begin{array}{l}\text { To explore how } \\
\text { transitioning from } \\
\text { compensatory care } \\
\text { to reablement care } \\
\text { is not merely } \\
\text { a practical process, } \\
\text { but also a deeply } \\
\text { normative one }\end{array}$ & $\begin{array}{l}\text { Ethnographic } \\
\text { field work }\end{array}$ & 8 weeks & $\begin{array}{l}\text { OTs } \\
\text { RNs } \\
\text { PTs } \\
\text { Allied health } \\
\text { personnel }\end{array}$ & $\begin{array}{l}\text { One municipality, } \\
\text { involving } 31 \text { older } \\
\text { people (of which } 8 \\
\text { received } \\
\text { reablement) } \\
\text { Interviews with } 13 \\
\text { HCPs }\end{array}$ & $\begin{array}{l}\text { Individualized } \\
\text { reablement } \\
\text { program includes } \\
\text { reablement training. } \\
\text { Allied health } \\
\text { personnel (upon } \\
\text { instruction by } \\
\text { health } \\
\text { professionals) } \\
\text { works to re-enable } \\
\text { the older person to } \\
\text { manage ADLs }\end{array}$ & $\begin{array}{l}\text { Experienced } \\
\text { transformation of } \\
\text { roles and mindsets } \\
\text { Values/knowledge of } \\
\text { PA influenced } \\
\text { practice }\end{array}$ \\
\hline $\begin{array}{l}\text { Baker et al, }{ }^{49} \\
200 I, \text { USA }\end{array}$ & $\begin{array}{l}\text { To describe the } \\
\text { development of } \\
\text { a restorative model } \\
\text { of home care } \\
\text { designed to } \\
\text { integrate medical } \\
\text { treatments for } \\
\text { acute conditions } \\
\text { with personal care } \\
\text { and rehabilitation } \\
\text { for chronic } \\
\text { disabilities in order } \\
\text { to improve older } \\
\text { adults' functional } \\
\text { outcomes }\end{array}$ & $\begin{array}{l}\text { Description of } \\
\text { design and } \\
\text { implementation } \\
\text { of a restorative } \\
\text { care model }\end{array}$ & $\begin{array}{l}\text { Mean } \\
\text { duration of } \\
\text { the } \\
\text { Restorative } \\
\text { care was } 24.8 \\
\text { days* }\end{array}$ & $\begin{array}{l}\text { Nurses } \\
\text { PTs } \\
\text { OT } \\
\text { Allied health } \\
\text { personnel }\end{array}$ & $\begin{array}{l}\text { Model development } \\
\text { within a branch of } \\
\text { a homecare agency. } \\
\text { Work group } \\
\text { included two } \\
\text { researchers (RN, } \\
\text { PT), two RNs, } 2 \\
\text { PTs, two allied } \\
\text { health personnel. } \\
\text { Staff and six older } \\
\text { adults participated } \\
\text { in focus groups }\end{array}$ & $\begin{array}{l}\text { Supporting older } \\
\text { adults to perform } \\
\text { activities } \\
\text { themselves. Help } \\
\text { patients safely } \\
\text { practice regaining } \\
\text { function. } \\
\text { Prevention of } \\
\text { sedentary behavior. } \\
\text { Allied health } \\
\text { personnel were } \\
\text { trained to help } \\
\text { patients follow } \\
\text { through on } \\
\text { prescribed } \\
\text { exercises and gait } \\
\text { and transfer } \\
\text { training }\end{array}$ & $\begin{array}{l}\text { Competencies of } \\
\text { allied health } \\
\text { personnel was } \\
\text { considered an } \\
\text { important factor. } \\
\text { Simple exercises } \\
\text { were considered } \\
\text { beneficial. } \\
\text { Values/knowledge of } \\
\text { PA influenced } \\
\text { practice }\end{array}$ \\
\hline
\end{tabular}

(Continued) 
Table 2 (Continued).

\begin{tabular}{|c|c|c|c|c|c|c|c|}
\hline Author & Aim & Method & $\begin{array}{l}\text { Duration of } \\
\text { Intervention }\end{array}$ & $\begin{array}{l}\text { HCPs } \\
\text { Involved }\end{array}$ & Informants & $\begin{array}{l}\text { PA } \\
\text { Characteristics }\end{array}$ & PA Experiences \\
\hline $\begin{array}{l}\text { Burton et al, }{ }^{47} \\
2014 \text {, Australia }\end{array}$ & $\begin{array}{l}\text { To determine } \\
\text { whether a lifestyle } \\
\text { and functional } \\
\text { exercise program } \\
\text { (LiFE) was suitable } \\
\text { for delivery in } \\
\text { a restorative home } \\
\text { care service }\end{array}$ & $\begin{array}{l}\text { Feasibility study; } \\
\text { Pilot } \\
\text { intervention } \\
\text { study, including } \\
\text { interviews with } \\
\text { clients and care } \\
\text { managers }\end{array}$ & $\begin{array}{l}\text { Average 7-8 } \\
\text { weeks }\end{array}$ & $\begin{array}{l}\text { RNs } \\
\text { OTs } \\
\text { PTs Aides* }\end{array}$ & 9 clients & $\begin{array}{l}\text { Exercises } \\
\text { incorporated into } \\
\text { daily activities. An } \\
\text { individually targeted } \\
\text { Lifestyle exercise } \\
\text { program as } \\
\text { intervention, } \\
\text { including strength } \\
\text { and balance } \\
\text { activities; } \\
\text { a standardized } \\
\text { exercise program as } \\
\text { comparative } \\
\text { intervention. Manual } \\
\text { delivered, including } \\
\text { guidance on how to } \\
\text { progress exercises }\end{array}$ & $\begin{array}{l}\text { Simple exercises } \\
\text { were considered } \\
\text { beneficial. Beneficial } \\
\text { with written } \\
\text { instructions }\end{array}$ \\
\hline $\begin{array}{l}\text { Liaanen et al, } \\
2019 \text {, Norway }\end{array}$ & $\begin{array}{l}\text { To provide } \\
\text { knowledge } \\
\text { regarding how } \\
\text { home care service } \\
\text { providers working } \\
\text { with reablement in } \\
\text { the home care } \\
\text { setting describe } \\
\text { their experiences }\end{array}$ & $\begin{array}{l}\text { Focus groups, } \\
\text { grounded } \\
\text { theory } \\
\text { approach }\end{array}$ & $\begin{array}{l}\text { Time-limited } \\
\text { within 6-8 } \\
\text { weeks, with } \\
\text { some } \\
\text { exceptions* }\end{array}$ & $\begin{array}{l}\text { OTs } \\
\text { PTs } \\
\text { RNs } \\
\text { Other home } \\
\text { care staff }\end{array}$ & $\begin{array}{l}25 \mathrm{HCPs} \text { (2 OTs, } \\
\text { II, RNs, } 4 \text { Health } \\
\text { workers, I } \\
\text { Specialist auxiliary } \\
\text { nurse, } 4 \text { auxiliary } \\
\text { nurses, I student } \\
\text { nurse, I Trainee } \\
\text { health worker and } \\
\text { I social educator) }\end{array}$ & Not reported & $\begin{array}{l}\text { HCPs experienced } \\
\text { a shift of care focus } \\
\text { from caring to } \\
\text { enabling. HCPs } \\
\text { emphasized that } \\
\text { reablement tended to } \\
\text { focus on activities of } \\
\text { daily living related to } \\
\text { personal hygiene and } \\
\text { food preparation, but } \\
\text { questioned a lack of } \\
\text { focus on outdoor and } \\
\text { social activities }\end{array}$ \\
\hline $\begin{array}{l}\text { Zingmark } \\
\text { et al, },^{33} 2020 \text {, } \\
\text { Sweden }\end{array}$ & $\begin{array}{l}\text { To explore the } \\
\text { characteristics and } \\
\text { differences of } \\
\text { occupational } \\
\text { therapy and } \\
\text { physiotherapy in } \\
\text { terms of focus } \\
\text { (what the } \\
\text { intervention was } \\
\text { aimed to improve), } \\
\text { content (what } \\
\text { actions were } \\
\text { implemented) and } \\
\text { duration within the } \\
\text { context of } \\
\text { reablement in } \\
\text { Swedish } \\
\text { municipalities }\end{array}$ & Surveys & $\begin{array}{l}\text { In most cases } \\
\text { the duration } \\
\text { was within } \\
\text { a period of } 6 \\
\text { weeks }\end{array}$ & $\begin{array}{l}\text { OTs } \\
\text { PTs } \\
\text { home care } \\
\text { staff } \\
\text { Collaboration } \\
\text { with other } \\
\text { professionals, } \\
\text { eg, district } \\
\text { nurses, social } \\
\text { workers, } \\
\text { managers }\end{array}$ & $\begin{array}{l}1393 \text { OTs } \\
1005 \text { PTs }\end{array}$ & $\begin{array}{l}\text { OTs focused mostly } \\
\text { on activities such as } \\
\text { walking indoors and } \\
\text { self-care. PTs } \\
\text { focused mainly on } \\
\text { walking indoors and } \\
\text { body function }\end{array}$ & Not reported \\
\hline
\end{tabular}

(Continued) 
Table 2 (Continued).

\begin{tabular}{|c|c|c|c|c|c|c|c|}
\hline Author & Aim & Method & $\begin{array}{l}\text { Duration of } \\
\text { Intervention }\end{array}$ & $\begin{array}{l}\text { HCPs } \\
\text { Involved }\end{array}$ & Informants & $\begin{array}{l}\text { PA } \\
\text { Characteristics }\end{array}$ & PA Experiences \\
\hline \multicolumn{8}{|c|}{ Perspectives of family members } \\
\hline $\begin{array}{l}\text { Hjelle et al, }{ }^{47} \\
2017, \text { Norway }\end{array}$ & $\begin{array}{l}\text { To explore and } \\
\text { describe how family } \\
\text { members } \\
\text { experience } \\
\text { participation in the } \\
\text { reablement process }\end{array}$ & $\begin{array}{l}\text { Qualitative; } \\
\text { Interviews (part } \\
\text { of larger } \\
\text { research } \\
\text { program; } \\
\text { Tuntland 2015) }\end{array}$ & Max 3 months & $\begin{array}{l}\text { OT } \\
\text { PT } \\
\text { Nurses } \\
\text { Allied health } \\
\text { personnel } \\
\text { Social } \\
\text { educator }\end{array}$ & $\begin{array}{l}\text { Six family members } \\
\text { (spouse, child or } \\
\text { other kinship) }\end{array}$ & $\begin{array}{l}\text { Same as Tuntland } \\
2015\end{array}$ & $\begin{array}{l}\text { Wanted information } \\
\text { about how to } \\
\text { support for PA. } \\
\text { Some relatives } \\
\text { missed follow-ups }\end{array}$ \\
\hline $\begin{array}{l}\text { Jakobsen } \\
\text { et al, }{ }^{51} 2019 \text {, } \\
\text { Norway }\end{array}$ & $\begin{array}{l}\text { To identify how } \\
\text { adult children } \\
\text { perceive the } \\
\text { collaboration } \\
\text { between older } \\
\text { parents, family } \\
\text { members, and } \\
\text { HCPs in } \\
\text { reablement services }\end{array}$ & $\begin{array}{l}\text { In-depth } \\
\text { interviews }\end{array}$ & 4-6 weeks & $\begin{array}{l}\text { PTs } \\
\text { OTs } \\
\text { RNs } \\
\text { Allied health } \\
\text { personnel }\end{array}$ & $\begin{array}{l}\text { Eight daughters, six } \\
\text { sons and } \\
\text { a daughter-in-law of } \\
\text { older adults } \\
\text { receiving } \\
\text { reablement }\end{array}$ & Not reported & $\begin{array}{l}\text { Some family } \\
\text { members wanted } \\
\text { information about } \\
\text { how to support for } \\
\text { PA. } \\
\text { Some family } \\
\text { members found this } \\
\text { responsibility } \\
\text { problematic. } \\
\text { Difficult to facilitate } \\
\text { PA as a relative }\end{array}$ \\
\hline
\end{tabular}

Note: *Information from personal contact with author.

Abbreviations: PA, physical activity; OT, occupational therapist; PT, physiotherapist; RN, registered nurses; ADL, activities of daily living.

activities, physical training, being active, or enhancing active engagement or independence in daily activities. Also, broader terms such as rehabilitation, occupational therapy, physiotherapy, or reablement intervention were used in contexts in which it was likely that some degree of PA was involved.

Encouragement of active engagement and practicing/ training ADL-tasks were explicitly reported as part of the reablement intervention in 10 of 15 intervention studies. ${ }^{26-31,33,34,36,39}$ These activities were related to the older adults' individual goals for reablement and could include activities such as indoor or outdoor mobility, dressing, bathing, kitchen activities, household activities, and social/leisure activities. However, in most studies it was not possible to capture if and to what degree/intensity the activity training involved PA. Only one (American) study mentioned sedentary behavior, and explicitly mentioned that the interventions were (among other aims) aimed at reducing sedentary behavior among participants. ${ }^{45}$

\section{Characteristics of Exercise Interventions}

Exercise interventions were reported to be included in 10 of the 15 intervention studies. Of these, seven studies reported only overall aims/characteristics of the exercises, such as "exercise programs targeting strength, balance or endurance". $27,28,3234-37$ One Australian intervention study, ${ }^{26}$ two Norwegian intervention studies including referrals to their study protocols, ${ }^{29,33,77,78}$ one Swedish quantitative study, ${ }^{49}$ and also one Norwegian field study (including four publications) $)^{53-55,66}$ reported additional characteristics of exercise interventions provided through reablement. Two different overall exercise approaches were described in these studies, including i) standardized exercise programs ${ }^{26,54}$ and ii) individually targeted/ adapted exercises. ${ }^{26,29,33}$

Burton et $\mathrm{al}^{26}$ was the only study that provided detailed descriptions of PA and exercise interventions incorporated in the reablement intervention. The aim of that study was to compare a lifestyle exercise program (LiFE) with a standardized structured exercise program in an Australian reablement setting. ${ }^{26,41}$ The LiFE program was aimed at improving balance, increasing strength, and preventing falls by embedding exercises into everyday activities. The program included 18 different exercises/ tasks (eg, knee bends, stair walking, tandem stand or walking, one leg stand; these were further specified in the article) that were incorporated into daily activities. 
The exercises were to be performed every day and did not require additional time. The control intervention of this study was a structured exercise program, which had been part of the restorative care services for years. The structured exercise program included eight prespecified balance and strength exercises (eg, sit to stand, stand and reach, toe taps) that the participants were asked to do in five repetitions three times a day (approximately 15-20 minutes per day).

In the two Norwegian intervention studies by Tuntland et $\mathrm{al}^{29,77}$ and Langeland et $\mathrm{al}^{33,77,78}$ it was described that daily training in activities was part of the general features of reablement, while exercise programs were recommended as individual features to improve strength, balance, or fine motor skills. In the studies by Eliassen et $\mathrm{al}^{53-55}$, they explored different perspectives of physiotherapy practice and supervision in reablement in Norwegian municipalities, and found that exercises were provided in all observed cases and that reablement plans contained elements of both exercises and daily activities. Though, while some of the teams mainly emphasized standardized exercises, other teams put more emphasis on daily activities and in some teams both exercises and practicing daily activities were integrated in their reablement approach. ${ }^{53,66}$ In a Swedish quantitative study by Zingmark et al, ${ }^{49}$ that investigated reablement intervention characteristics reported by 1395 OTs and 1006 PTs, they found that OTs mostly focused on activities such as walking indoors and self-care, while PTs mostly focused on walking indoors and activities aimed at improving body function (ie, strength, balance, range of motion). Activities related to walking outdoors were prioritized by OTs in $24 \%$ of reported cases, and by PTs in $38 \%$ of reported cases. ${ }^{49}$

The frequency for follow-up of exercises by HCPs was different between studies. Burton et $\mathrm{al}^{26}$ described that health professionals provided an average of three visits to participants, and that the participants were instructed to do the exercises unsupervised. At the visits in that study, the health professionals described the different exercises, discussed with the participants how they could incorporate the exercises into their daily routines (for the LiFE program), and provided support and encouragement for doing the exercises as well as other areas of their reablement. Tuntland et $\mathrm{al}^{29}$ and Langeland et $\mathrm{al}^{33}$ reported more frequent visits and described that HCPs would be present during daily training to build confidence, relearn skills, and stimulate the participant in self-management and selftraining. They also reported that the participants would be encouraged to perform exercise programs on their own.
The duration of the exercise interventions in Burton et $\mathrm{al}^{26}$ was 8 weeks (with care manager assistance), while Langeland et $\mathrm{al}^{33}$ reported a maximum of 10 weeks duration (average 5.7 weeks) and Tuntland et $\mathrm{al}^{29}$ a maximum of 3 months (with an average of 10 weeks) duration of the reablement intervention. Some of the included studies reported that written and/or illustrative manuals of the exercises/training was provided. ${ }^{26,29,43,54}$ None of the studies reported using equipment for PA/Exercise. No studies reported any intensity levels of physical activity interventions or exercises.

\section{Approaches Used to Target and Progress PA Interventions According to Individual Needs}

In some of the studies, it was reported that exercises were provided to reablement users if they had an individual need for this. ${ }^{26,29}$ However, there was little information about how the individual needs for exercises or PA were assessed. In the LiFE RCT study by Burton et $\mathrm{al}^{26}$ only older adults that had been prescribed an exercise intervention by their care manager (health professionals, including PTs, OTs, or RNs) were included in the study. Of the entire group receiving reablement, only $5.4 \%$ met the eligibility criteria of the study; one of which included that they had been referred to an exercise program. This could indicate that only a minority of reablement receivers in that setting were considered to benefit from participating in an exercise program. In a questionnaire study undertaken in the same state of Australia as the LiFE RCT, $30 \%$ of reablement clients recalled being given an activity program, and a third of them reported having been encouraged to be more physically active. ${ }^{46}$

Several of the studies reported that exercises were progressed and adapted during the reablement period according to the older adults' development of function. ${ }^{26,28,31,43,53}$ In the LiFE program and the structured exercise program in the study by Burton et al, ${ }^{26}$ progression of exercises was reported to be included, but it was not described how the need for progression was assessed. In the structured exercise program, it was reported that the participants were to progress to level two exercises on the back of the exercise sheet. In the RCT study by Hattori et al, ${ }^{31}$ rehabilitation specialists reviewed participants' goals in every module, in order to monitor their progress, assess their physical activities and training, and encourage behavioral changes, using an assessment sheet for self-management. In the field study by Eliassen et $\mathrm{al}^{53-55}$ the researchers observed that the 
characteristics of the targeting processes of the exercises were mainly divided into two groups; i) standardized approaches and ii) individually tailored approaches. In the standardized approaches, allied health personnel conducted training sessions based on standardized exercise programs. In these cases the reablement plan (including the exercises) was provided by the PTs, and the allied health personnel made minimal adjustments to this. In the second group, Individually tailored approaches targeting quality of movement, the exercises were individually targeted based on extended examinations and assessments by PTs, including functional analysis regarding movement and structure of muscles and joints, in addition to standardized physical performance tests. In these teams, the allied health personnel were instructed about movement quality, however it was described that only a few of them were able to pay attention to the small details of the instructions. ${ }^{54}$ Similar to this second approach, Moe and Brinchmann ${ }^{71}$ reported from another Norwegian field study that exercises (and other therapeutic activities) were based on a detailed screening that identified activity goals and functional impairments, as well as other factors contributing to functional loss such as pain, malnutrition, and medication use. Several of the studies described that the role of the health professionals was to be a consultant and/or advisor, including developing and adjusting a rehabilitation plan and supervising allied health personnel. The allied health personnel were then responsible for following up on the training, including, eg, encouraging, supporting, and ensuring security when the older adult performed everyday activities and/or exercises. . $^{53,54,58,62,66}$

\section{Compliance of PA Recommendations}

Burton et $\mathrm{a}^{26}$ was the only study that had assessed compliance with exercises during the intervention period, by using an exercise adherence diary. They found that participants undertook exercises on average 4.91-times a week (in the LiFE group) and 4.42-times a week (in the structured exercise group). In the 6-month follow-up study it was reported that the participants in both groups still undertook exercises, though a little less often (average of 3.45 times per week). ${ }^{41}$

\section{Q2: Experiences and Barriers for PA Older Adults' Perspectives}

Seven qualitative studies explored older adults' experiences of participating in reablement, ${ }^{67-73}$ of which four qualitative studies (three Norwegian and one from the
UK) $)^{69,71-73}$ and also one mixed method study ${ }^{44}$ touched upon themes related to PA, which are summarized in the following. Additionally, one Australian mixed-method study investigated motivators and barriers to being physically active for older people $\left(70^{+}\right)$that previously had received either reablement or usual home care services, ${ }^{42}$ and one Norwegian quantitative study explored which occupations and rehabilitation goals older people prioritized in a reablement setting. ${ }^{47}$

In the studies by Moe and Brinchmann ${ }^{71}$ and Magne and $\mathrm{Vik},{ }^{73}$ the older adults described how they experienced physical strengthening to be essential for their progress and that physical strengthening also led to increased participation in other activities in their daily life. Some older adults reported that they felt insecure when participating in activities, due to fear of injuries or fear of falling, ${ }^{71,73}$ and that the support from the reablement team helped them gain a sense of security and confidence when performing daily activities. ${ }^{71,73}$ Similarly, Hjelle et al ${ }^{69}$ found that the older adults' willpower to engage in exercises and everyday activities evolved during their recovery. The older adults' determination and willpower was considered important for their engagement in exercise and performing everyday activities. ${ }^{69}$ However, some older adults found the exercises to be too easy and not inspiring. ${ }^{73}$

Encouragement, support, supervision, and a push by reablement staff was considered a motivational factor for increasing PA. ${ }^{69,71}$ The support from the reablement staff stimulated some older adults to do exercises/activities on their own and also to continue PA after the reablement period, while others were only motivated when the staff were encouraging them. ${ }^{69}$ Older adults reported that they preferred to plan their own day themselves, including deciding when to perform training and activities, and that being in their home environment stimulated them to be independent and take part in everyday activities. ${ }^{69}$ Additionally, the older adults' social network was considered an important factor to enable active living and participating in daily activities. ${ }^{73}$ Organizing the home to make it safer and easier to maneuver inside, as well as reducing barriers for outside activities were also reported as important for activity performance. ${ }^{71}$

Some of the older adults expressed that they considered exercising or training to be something different than practicing activities. ${ }^{69}$ They considered training in reablement as doing physical exercises in order to improve physical strength, balance, or range of motion, but they did not consider ADL as training. In a study from the UK by 
Wilde and Glendinning, ${ }^{72}$ service users expressed frustration at the limited access to wider sources of professional expertise (social workers and OTs were involved in that setting), particularly with the aim of maintaining or improving their ability to walk outdoors and manage stairs so that they could participate in social activities. Likewise, another UK study reported that outdoor mobility goals were difficult to reach due to fluctuations in the users' health or weather conditions. ${ }^{44}$

Tuntland et $\mathrm{al}^{29}$ investigated what types of activities or tasks that older adults that had participated in an Norwegian RCT reported as difficult to perform, and which activities they prioritized as rehabilitation goals. ${ }^{47}$ Functional mobility goals, such as going for a walk, walking up stairs, transferring or outdoor mobility were most frequently prioritized ( $35 \%$ of prioritized sub-areas), followed by personal care activities, such as taking a shower or dress/undress ( $18 \%$ of prioritized sub-areas) and household activities, such as preparing food or cleaning/ vacuuming the house ( $15 \%$ of prioritized sub-areas). They also reported that some of the responses remained unclassified (3.5\%) because they were mainly impairmentbased goals such as improving balance, strength, or memory, rather than activity-based goals.

Burton et $\mathrm{al}^{42}$ found in their mixed-method study that health and fitness (reported by $56.3 \%$ of reablement receivers) and well-being (55.3\%) were the top two reasons participants gave for being active, followed by enjoyment (48.4\%), social/family (44.7\%), transport (20\%), weight loss $(18.6 \%)$, walking the dog $(11.6 \%)$, and competition/challenge $(7 \%)$. The highest ranked barriers were ongoing injury/illness (reported by $45.6 \%$ of reablement receivers) and feeling too old (41.4\%), followed by temporary injury/ illness $(17.7 \%)$, nobody to be physically active with (12.1\%), lack of transport (11.6\%), cost (7\%), nowhere to be physically active (4.7\%), not interested (3.7), do not know how to be physically active (1.9\%), and lack of time (3.7\%).

\section{HCPs' Perspectives}

Eighteen qualitative studies, one feasibility study, and one implementation study investigated inquiries based on HCPs experiences or perspectives on reablement, ${ }^{43,45,50-66}$ but none of these specifically aimed at investigating or exploring HCPs' experiences related to PA facilitation. However, some of the studies - of which seven were Norwegian, ${ }^{53-55,58,60,65,66}$ two were Danish, ${ }^{51,62}$ one Australian, ${ }^{43}$ and one from the US, ${ }^{45}$ brought up perspectives from HCPs related to activity training or exercises, which are presented in the following.
HCPs considered the organization of tasks between health professionals and allied health personnel to be beneficial for reaching out to a larger population and for giving more intensive training. ${ }^{54,58}$ However, it was also reported that the competencies of the allied health personnel and the team collaboration could have an impact on the content of the training or exercises. ${ }^{45,53-55} \mathrm{HCPs}$ in several of the studies noted that it was advantageous to implement simple and recognizable exercises that could easily be explained to both the allied health personnel and the older adults. ${ }^{43,45,54}$ It was considered beneficial to use written instructions for the exercises/training, ${ }^{43,45}$ and in one study they reported lower compliance among users when, eg, giving complicated verbal instructions without leaving written instructions. ${ }^{45}$

In some reablement settings, the ability to target the exercises/training to the older adults' individual needs, including a focus on movement quality, was more emphasized than standardized exercise programs. ${ }^{53}$ In these teams, a more intense collaboration between healthcare professionals (PTs in this case) and allied healthcare personnel was observed, including both formal and informal meeting-points, as well as on-going supervision and common reflection in the team. It was emphasized that allied healthcare personnel had the required competencies to follow-up individually targeted interventions, that they were capable of independent evaluations of the older adults' function and independence during the period, and also that they had sufficient competence to evaluate the need for additional therapeutic assistance. ${ }^{53}$ The allied healthcare personnel in these teams expressed that it was difficult to point out what to look for, but that they learned along the way. Thus, this approach relied more on building the competencies of allied health personnel, which was reported as a limitation in other settings. ${ }^{45,54}$ Some HCPs suggested reablement was not sufficiently targeted towards outdoor activities, such as going to social activities or going grocery shopping. ${ }^{65}$

The roles of the allied healthcare personnel were found to be transformed from being carers to becoming trainers, and implied a change of mindset of what it means to be a good carer. ${ }^{51}$ However, this transformation of mindset could also lead to discrepancies regarding different disciplinary views and norms related to caring and rehabilitation. ${ }^{51}$ Also, some HCPs experienced an ambivalence related to ensuring a good balance between helping and enabling the older adults to perform activities. ${ }^{65}$ Phrases such as keeping your hands behind your back 
and do not take over for the citizen were reported to be commonly repeated in a Danish reablement setting, and HCPs were reported to increasingly assume a physically passive position, including a more distanced, observational, and instructing practice. ${ }^{62}$

One study investigated HCPs perspectives regarding family members of older adults in a reablement setting. ${ }^{60}$ They found that family members were sometimes considered a resource, that could facilitate the older adult to participate in additional activities. However, the family members could also be a barrier to (physical) activity, by taking over the older adults daily activities. Several studies reported that the knowledge and values related to the benefits of PA and active aging sometimes were met with skepticism or resistance from older adults themselves, family members, HCPs, or by habitual traditions of running healthcare services. ${ }^{45,51,60,64}$

\section{Family Members' Perspectives}

Three Norwegian studies had investigated family members' perspectives (including relatives, adult children, and caregivers/spouses) and their experiences with reablement ${ }^{71,74,75}$ and two of them touched upon some themes related to PA. ${ }^{74,75}$ Family members expressed that they wanted information about how to support and motivate the older adult to engage in PA. ${ }^{74,75}$ However, some of them expressed that taking this responsibility was problematic. ${ }^{75}$ Some of the family members perceived that it was difficult for them, in the role as a family member, to facilitate PA, and that the older adult (their mother/father, etc.) was more likely to listen to PA advice from the reablement staff. ${ }^{75}$ Some of the family members missed follow-ups, including motivation to train and practice to ensure that the older adults' achieved function was maintained after the reablement was finished. ${ }^{74}$

\section{Q3: Assessment of Physical Fitness and PA Levels Physical Fitness}

Five of the 15 intervention studies that were included (three Australian studies and two Norwegian studies) reported using at least one standardized clinical measure of physical fitness. ${ }^{26,27,29,33,34}$ Timed up and Go (TUG) was most frequently used $(n=4)$, with the aim of measuring functional mobility. ${ }^{26,27,29,34}$ One study used the Short Physical Performance battery (SPPB) to measure lower extremity strength, walking speed, and static balance. ${ }^{33}$
Specific strength assessments included Sit-to-stand one repetition and five repetitions ${ }^{26}$ and Grip Strength/ Dynamometer, ${ }^{29}$ while specific balance assessments included Functional reach/static balance ${ }^{26}$ and Tandem walk/dynamic balance. ${ }^{26}$ Follow-up measures of physical fitness in the intervention studies were made at 8 weeks, ${ }^{26}$ 10 weeks, ${ }^{33} 3$ months, ${ }^{27,29,34} 6$ months, ${ }^{33,41} 9$ months, ${ }^{29}$ and 12 months. ${ }^{27,33,34}$ Eliassen et $\mathrm{al}^{54}$ reported in their field study that SPPB was used as a standard assessment method in all of the seven included Norwegian municipalities, and that some of the municipalities also used additional tests (no further details provided) related to movement quality. Zingmark et $\mathrm{al}^{49}$ reported variable use of standardized clinical measures of physical fitness in Swedish reablement settings, including a range of different assessment methods such as the 30s chair stand test, TUG and 10 meter walking test.

Among the included RCT-studies, the two studies that reported mobility outcomes (TUG) did not have comparable comparison interventions (one compared two different exercise interventions in reablement and the other compared reablement with standard homecare services). ${ }^{26,29}$ Thus, a synthesis of this evidence would not be considered adequate. Among the non-RCTs, more positive results for physical fitness outcomes were reported (for TUG and SPPB), however the design of these studies meant that the risk of bias would be too high to be included in a synthesis of outcomes. No other outcome measures related to physical fitness were comparable in the RCTs. Overall, the only significant differences related to physical fitness in an RCT study were reported by Burton et $\mathrm{al}^{26}{ }^{26}$ who reported significantly better outcomes in balance (tandem walk) in the reablement + LiFE program compared to reablement + structured exercise program.

\section{Physical Activity}

None of the intervention studies assessed levels of PA or sedentary behavior. However, one Australian RCT reported using an exercise diary to assess adherence. ${ }^{26}$ One feasibility study ${ }^{43}$ used the physical activity scale for the elderly (PASE) to assess habitual PA among older adults receiving reablement in an Australian setting. They also used an accelerometer to assess energy expended over 7 days. It was, however, decided not to include accelerometer assessments in the following RCT because of poor compliance and potential for causing discomfort to some participants. $^{43}$ 
The only study that reported PA levels among older adults (that previously had received either reablement or usual care), was an Australian questionnaire study ( $\mathrm{n}=506$ ) that used the self-reported PASE questionnaire to assess and compare PA levels between participants receiving reablement and usual care. ${ }^{46}$ They found that $77.7 \%$ of all respondents reported that they were physically active for the recommended minimum 30 minutes of moderate exercise each day, and that there were insignificant differences between groups. ${ }^{46}$

\section{Discussion}

We conducted a systematic scoping review with the aim of mapping existing evidence of how PA strategies have been integrated and explored in reablement research and to identify knowledge gaps. We identified and mapped evidence of how PA strategies have been integrated and explored in 51 studies of reablement. The review revealed that PA recommendations, such as the WHO recommendations, ${ }^{79}$ were rarely mentioned, and that the degree and intensity of PA or inactivity/sedentary behavior among older adults' receiving reablement has been given little attention in research.

The review showed that there was little information regarding the causes of functional decline among older adults receiving reablement. Baseline measures of physical fitness in the identified intervention studies indicated that the older adults in general had reduced physical fitness, which is likely to be part of the causes of functional decline. ${ }^{26,27,29,33,34}$ PA levels have been found to correlate with physical fitness among older adults in other settings, ${ }^{12}$ but none of the identified intervention studies in this review included information about the PA levels among older adults in receipt of reablement.

Functional mobility, such as walking, stair walking, transferring, or outdoor mobility was reported as commonly prioritized goals among older adults receiving reablement. ${ }^{47}$ These types of activities are premises for participating in a large range of daily activities, both indoor, outdoor, and social activities. It was reported that older adults participating in reablement perceived that improvements of functional mobility or physical strengthening lead to increased participation in other activities, increased self-confidence, and helped them to increase the freedom to plan their daily activities themselves. ${ }^{69,71}$ This may suggest that a focus on improving basic functional mobility and physical fitness is essential both for achieving the individual goals of the older adults, as well as for increasing general activity levels and participation. However, older adults' experiences of PA in a reablement setting should be further explored.

The older adults' individual goals were reported by HCPs as crucial to their development of a reablement plan. ${ }^{50,56,59,61,64}$ Although facilitation of activity through practicing daily activities or enhancing participation in daily activities seem to be a central component of reablement, the degree of PA involved in these activities may vary considerably, depending on the activities involved and the functional levels of the older adult. Thus, there may be large differences to the degree of PA involved in reablement. None of the studies reported any measures of intensity of PA or exercises in the reablement interventions. This prohibits the possibility for recommending effective exercise/PA intensity levels for use in reablement. Also, there is limited evidence on the progression of exercises over a period of reablement. More evidence is required to understand what level and when progressions should be applied to gain maximum effect for older people receiving reablement. An increased focus on PA behavior may be helpful in order to improve the older adults' achievement of their goals and also for maintenance of function after reablement. It should be further explored if PA recommendations such as the ones recommended by WHO are feasible and effective in a reablement setting, and also what type of support the older adults' need in order to maintain PA and function after reablement.

Several of the included studies reported that societal expectations regarding aging and activity influenced PA facilitation in this setting, both from the older adults themselves, family members, and HCPs. ${ }^{45,51,60,64}$ HCPs should be aware of such existing assumptions when informing about PA. It was also reported that the older adults' motivation and confidence related to PA increased along with their experiences of PA and improvement of function. ${ }^{69,71}$ This is in line with findings in other settings, where older adults perceive that the value and enjoyment of being physically active are important factors for participating in PA interventions, and that positive PA experiences increase their motivation of PA. ${ }^{80}$ Thus, the HCPs may need to adapt their motivational strategies continually during the reablement period in order to facilitate PA as part of the reablement intervention.

Although exercises often were reported as a component of reablement, the characteristics of these interventions were in general poorly reported and it was unclear how HCPs made judgments and recommendations regarding PA and exercises. 
One of the priority areas of the WHO within their physical activity strategy for 2016-2025 is to improve the quality of advice on PA by HCPs to older people. ${ }^{18}$ Reablement may be a convenient setting for integrating and developing such recommendations, due to its features of being personcentered and intensive. ${ }^{80}$ There is a need to further investigate how HCPs can facilitate PA in a reablement setting in an effective way. Based on the findings from this scoping review, we suggest that the following gaps of knowledge are important to address in future research.

\section{Gaps of Knowledge}

- To what degree is reduced physical fitness part of the causes of functional decline among older adults in receipt of reablement?

- To what degree are older adults in receipt of reablement physically active?

- How do older adults' experience PA facilitation in a reablement setting?

- What type(s) of PA recommendations and exercises are feasible and effective in a reablement setting?

- How does the context of reablement influence PA facilitation?

- What knowledge and competencies are needed by HCPs in order to facilitate PA in reablement?

- How should HCPs appropriately balance and prioritize evidence-based knowledge of PA with the person-centered focus in reablement?

\section{Suggestions for Future Research}

We suggest that future studies of reablement interventions ensure that the interventions are explicitly described, including detailed characteristics of content, intensity, duration, and delivery of the interventions. Due to the patient-centered and - to some degree - unpredictable nature of reablement interventions, we also suggest that intervention studies not only include a pre-planned description of the intervention, but also include reports or measures of the content of the interventions that was actually given throughout the intervention period. We further suggest that the research of reablement interventions pay further attention towards exploring and investigating the effectiveness of more specific components of reablement, such as PA components. This should also include exploration of older adults', HCPs', and - when appropriate - relatives' perspectives and experiences with PA in order to identify potential facilitators and barriers.
This scoping review reveals a large divergency in the delivery of reablement interventions, which is likely to be affected by many factors, such as differences in national and local healthcare policies, health professionals involved in reablement, or available resources in reablement. We suggest that - rather than seeing this divergency as a bias to reablement research - that these contextual divergencies are recognized and further explored in reablement research in order to identify facilitators and barriers for successful reablement delivery.

We also suggest that future studies include additional data of the population group related to causes of functional decline within this population, including (but not limited to) measures of physical fitness and measures of physical activity behavior. We recommend that research is aimed and designed towards identifying subgroups of people that may receive particular benefit (or lack of benefit) of reablement or of particular components of reablement. Furthermore, there is a need to point out feasible measurement instruments that are relevant to this population and can be used more consistently in order to improve comparability between studies. We suggest that specific, clinical outcome measures are used as supplementary measures in order to identify adjustable factors that may be of particular significance for improvement of function.

Finally, we suggest that further research of reablement should aim to explore what type of knowledge and competencies are needed by HCPs in order to provide effective evidence-based and person-centered reablement.

\section{Strengths and Limitations}

A strength of this scoping review was the comprehensive systematic search strategy aiming to identify a broad range of study designs related to the topic. We also followed acknowledged method recommendations for scoping reviews and did duplicate study selection and data extraction in order to raise validity.

A limitation to the study is that the eligibility criteria that we used may not have captured all types of reablement interventions, due to the variability of reablement characteristics. However, by building on a reablement definition used by one of the latest systematic reviews in the field, ${ }^{4}$ we aimed to capture the main essence of reablement research. Since PA in general was vaguely described and defined in the included studies, the extraction of data related to PA experiences were based on the reviewers' discretion, which can be a limitation to the reliability of the study findings. However, by pilot- 
testing the data extraction forms and performing data extraction in duplicate, it was enhanced to optimize the consistency of this process. Another limitation to the study is that we did not use search strategies with other search terms than English, and we may have missed eligible studies in the other languages that we intended to include. Although the time-limitation used in the search strategy may seem a limitation to the study, our findings strengthened our anticipation that the main body of literature was published in the latest part of the last two decades. This scoping review did not consider the quality of the included studies, and thus it was not possible (or intended) to synthesize and evaluate research evidence. Rather, the intention was to identify and map the current evidence in order to identify gaps of knowledge for future research.

\section{Conclusion}

There is limited evidence of how PA is integrated in reablement, including how PA strategies are targeted to older adults' individual needs and preferences in a reablement setting. Also, there is a lack of understanding concerning the knowledge and competencies that are required by HCPs in order to facilitate PA among older adults receiving reablement. While PA levels are known to be highly related to older adults' physical fitness and function in other settings, there is limited evidence regarding how reablement influences PA levels and physical fitness. None of the reablement studies reported measures that informed (changes of) PA levels during reablement. Although some measures of physical fitness were reported, the use of measurement instruments between studies were inconsistent and it should be further considered which instruments are most appropriate in a reablement setting. Further research is needed to explore how PA should be integrated in reablement and how it should be prioritized among other intervention components included in reablement.

\section{Abbreviations}

PA, physical activity; HCP, healthcare providers; PT, physiotherapist; OT, occupational therapist; RN, registered nurse; TUG, timed up and go; SPPB, short physical performance battery; STS, sit-to-stand; PASE, Physical Activity Scale for the Elderly; RCT, randomized controlled trial.

\section{Data Sharing Statement}

All data generated or analyzed during this study are included in this published article and its Supplementary $\underline{\text { Files } 1}$ and $\underline{2}$.

\section{Acknowledgments}

We thank Marit Veie, specialist librarian at Nord University, Norway, for guidance in the development of the search strategy and assistance with performing searches in scientific databases.

\section{Author Contributions}

HLM carried out searches (in cooperation with a librarian), did study selection, data extraction, mapping of results, and made a draft of the manuscript. CFM carried out duplicate study selection and data extraction. EB and LU were involved to solve discrepancies between reviewers. All authors contributed to data analysis, drafting, or revising the article, have agreed on the journal to which the article will be submitted, gave final approval of the version to be published, and agree to be accountable for all aspects of the work.

\section{Funding}

This study is part of a $\mathrm{PhD}$ project undertaken by the first author, HLM, and funded by Nord University, Norway. The funders had no role in study design, data collection and analysis, decision to publish, or preparation of the manuscript.

\section{Disclosure}

None of the authors participating in the review (HLM, CFM, EB, and LU) have any competing interests.

\section{References}

1. Whitehead PJ, Worthington EJ, Parry RH, Walker MF, Drummond AE. Interventions to reduce dependency in personal activities of daily living in community dwelling adults who use homecare services: a systematic review. Clin Rehabil. 2015;29(11):1064-1076. doi:10.1177/0269215514564894

2. Tessier A, Beaulieu MD, McGinn CA, Latulippe R. Effectiveness of reablement: a systematic review. Healthcare policy $=$ Politiques de sante. 2016;11(4):49-59. doi:10.12927/hcpol.2016.24594

3. Legg L, Gladman J, Drummond A, Davidson A. A systematic review of the evidence on home care reablement services. Clin Rehabil. 2016;30(8):741-749. doi:10.1177/0269215515603220

4. Cochrane A, Furlong M, McGilloway S, Molloy DW, Stevenson M, Donnelly M. Time-limited home-care reablement services for maintaining and improving the functional independence of older adults. Cochrane Database Syst Rev. 2016;10:Cd010825.

5. Pettersson C, Iwarsson S. Evidence-based interventions involving occupational therapists are needed in reablement for older community-living people: a systematic review. Br J Occup Ther. 2017;80(5):273-285. doi:10.1177/0308022617691537

6. World Health Organization. Global strategy and action plan on ageing and health. Geneva; 2017. Available from: https://www.who.int/age ing/WHO-GSAP-2017.pdf. Accessed September 30, 2020. 
7. Sims-Gould J, Tong CE, Wallis-Mayer L, Ashe MC. Reablement, reactivation, rehabilitation and restorative interventions with older adults in receipt of home care: a systematic review. J Am Med Dir Assoc. 2017;18 (8):653-663. doi:10.1016/j.jamda.2016.12.070

8. World Health Organization. Global recommendations on physical activity for health. Geneva; 2010. Available from: https://www.who. int/dietphysicalactivity/publications/9789241599979/en/. Accessed September 30, 2020.

9. World Health Organization. Physical activity [Internet]. Available from: http://www.who.int/ncds/prevention/physical-activity/en/. Accessed October 10, 2019.

10. Caspersen CJ, Powell KE, Christenson GM. Physical activity, exercise, and physical fitness: definitions and distinctions for health-related research. Public Health Rep. 1985;100(2):126-131.

11. Tremblay MS, Aubert S, Barnes JD, et al. Sedentary behavior research network (SBRN) - terminology consensus project process and outcome. Int J Behav Nutr Phys Act. 2017;14(1):75. doi:10.1186/ s12966-017-0525-8

12. Stessman J, Hammerman-Rozenberg R, Cohen A, Ein-Mor E, Jacobs JM. Physical activity, function, and longevity among the very old. JAMA Intern Med. 2009;169(16):1476-1483.

13. El-Khoury F, Cassou B, Charles MA, Dargent-Molina P. The effect of fall prevention exercise programmes on fall induced injuries in community dwelling older adults: systematic review and meta-analysis of randomised controlled trials. BMJ. 2013;347:f6234

14. Lozano-Montoya I, Correa-Perez A, Abraha I, et al. Nonpharmacological interventions to treat physical frailty and sarcopenia in older patients: a systematic overview - the SENATOR project ONTOP series. Clin Interv Aging. 2017;12:721-740. doi:10.2147/CIA.S132496

15. Chou $\mathrm{CH}$, Hwang CL, Wu YT. Effect of exercise on physical function, daily living activities, and quality of life in the frail older adults: a meta-analysis. Arch Phys Med Rehabil. 2012;93(2):237-244. doi:10.1016/j.apmr.2011.08.042

16. Baert V, Gorus E, Mets T, Geerts C, Bautmans I. Motivators and barriers for physical activity in the oldest old: a systematic review. Ageing Res Rev. 2011;10(4):464-474. doi:10.1016/j.arr.2011.04.001

17. Burton E, Farrier K, Lewin G, et al. Motivators and barriers for older people participating in resistance training: a systematic review. J Aging Phys Act. 2017;25(2):311-324. doi:10.1123/japa.2015-0289

18. World Health Organization. Physical activity strategy for the WHO European Region 2016-2025. Regional office for Europe 2016. Available from: http://www.euro.who.int/_ data/assets/pdf_file/0014/ 311360/Physical-activity-strategy-2016-2025.pdf?ua=1. Accessed September 30, 2020.

19. Olanrewaju O, Kelly S, Cowan A, Brayne C, Lafortune L. Physical activity in community dwelling older people: a systematic review of reviews of interventions and context. PLoS One. 2016;11(12): e0168614. doi:10.1371/journal.pone.0168614

20. Peters MDJ, Godfrey C, McInerney P, Munn Z, Tricco AC, Khalil, H. Chapter 11: Scoping Reviews (2020 version). In: Aromataris E, Munn Z (Editors). JBI Manual for Evidence Synthesis, JBI. 2020. Available from: https://synthesismanual.jbi.global. doi:10.46658/ JBIMES-20-12

21. Peters MD, Godfrey CM, Khalil H, McInerney P, Parker D, Soares CB. Guidance for conducting systematic scoping reviews. Int $J$ Evid Based Healthc. 2015;13(3):141-146. doi:10.1097/ XEB.0000000000000050

22. Tricco AC, Lillie E, Zarin W, et al. PRISMA extension for scoping reviews (PRISMA-ScR): checklist and explanation. Ann Intern Med. 2018;169(7):467-473. doi:10.7326/M18-0850

23. Mjøsund HL, Burton E, Moe CF, Uhrenfeldt L. Integration of physical activity in reablement for community-dwelling older adults: a scoping review protocol. JBI Database System Rev Implement Rep. 2019;17(9):1924-1932. doi:10.11124/JBISRIR-2017-003938
24. Ryburn B, Wells Y, Foreman P. Enabling independence: restorative approaches to home care provision for frail older adults. Health Soc Care Community. 2009;17(3):225-234. doi:10.1111/j.1365-2524. 2008.00809.x

25. Ouzzani M, Hammady H, Fedorowicz Z, Elmagarmid A. Rayyan a web and mobile app for systematic reviews. Syst Rev. 2016;5(210). doi:10.1186/s13643-016-0384-4

26. Burton E, Lewin G, Clemson L, Boldy D. Effectiveness of a lifestyle exercise program for older people receiving a restorative home care service: a pragmatic randomized controlled trial. Clin Interv Aging. 2013;8:1591-1601. doi:10.2147/CIA.S44614

27. Lewin G, De San Miguel K, Knuiman M, et al. A randomised controlled trial of the Home Independence Program, an Australian restorative home-care programme for older adults. Health Soc Care Community. 2013;21(1):69-78. doi:10.1111/j.1365-2524.2012.01088.x

28. Parsons M, Parsons J, Rouse P, et al. Supported Discharge Teams for older people in hospital acute care: a randomised controlled trial. Age Ageing. 2018;47(2):288-294. doi:10.1093/ageing/afx169

29. Tuntland H, Aaslund MK, Espehaug B, Førland O, Kjeken I. Reablement in community-dwelling older adults: a randomised controlled trial. BMC Geriatr. 2015;15:145. doi:10.1186/s12877-0150142-9

30. Whitehead PJ, Walker MF, Parry RH, Latif Z, McGeorge ID, Drummond AER. Occupational therapy in homecare re-ablement services (OTHERS): results of a feasibility randomised controlled trial. BMJ Open. 2016;6(8):e011868. doi:10.1136/bmjopen-2016011868

31. Hattori S, Yoshida T, Okumura Y, Kondo K. Effects of reablement on the independence of community-dwelling older adults with mild disability: a randomized controlled trial. Int J Environ Res Public Health. 2019;16(20):3954. doi:10.3390/ijerph16203954

32. Jeon YH, Krein L, Simpson JM, et al. Feasibility and potential effects of interdisciplinary home-based reablement program (I-HARP) for people with cognitive and functional decline: a pilot trial. Aging Ment Health. 2019:1-10. doi:10.1080/13607863.2019.1642298

33. Langeland E, Tuntland H, Folkestad B, Førland O, Jacobsen FF, Kjeken I. A multicenter investigation of reablement in Norway: a clinical controlled trial. BMC Geriatr. 2019;19(1):29. doi:10.1186/ s12877-019-1038-X

34. Lewin G, Ermeulen S. A non-randomised controlled trial of the Home Independence Program (HIP): an Australian restorative programme for older home-care clients. Health Soc Care Community. 2010;18(1):91-99.

35. Tinetti ME, Baker D, Gallo WT, et al. Evaluation of restorative care vs usual care for older adults receiving an acute episode of home care. JAMA. 2002;287(16):2098-2154. doi:10.1001/jama.287.16.2098

36. Tinetti ME, Charpentier P, Gottschalk M, Baker DI. Effect of a restorative model of posthospital home care on hospital readmissions. J Am Geriatr Soc. 2012;60(8):1521-1526. doi:10. 1111/j.1532-5415.2012.04060.x

37. Whitney SL, Marchetti GF, Ellis JL, Otis L. Outcomes of usual versus a specialized falls and balance program in the home. Home Healthc Now. 2015;33(5):265-274. doi:10.1097/NHH.0000000000000232

38. Slater P, Hasson F. An evaluation of the reablement service programme on physical ability, care needs and care plan packages. J Integr Care. 2018;26(2):140-149. doi:10.1108/JICA-11-2017-0042

39. Winkel A, Langberg H, Wæhrens EE. Reablement in a community setting. Disabil Rehabil. 2015;37(15):1347-1352.

40. Beresford B, Mayhew E, Duarte A, et al. Outcomes of reablement and their measurement: findings from an evaluation of English reablement services. Health Soc Care Community. 2019;27 (6):1438-1450. doi:10.1111/hsc. 12814

41. Burton E, Lewin G, Clemson L, Boldy D. Long-term benefits of a lifestyle exercise program for older people receiving a restorative home care service: a pragmatic randomized controlled trial. Healthy Aging Clin Care Elder. 2014;6:1-9. 
42. Burton E, Lewin G, Boldy D. Barriers and motivators to being physically active for older home care clients. Phys Occup Ther Geriatr. 2013;31(1):21-36. doi:10.3109/02703181.2012.751474

43. Burton E, Lewin G, Clemson L, Boldy D. Determining the feasibility of a lifestyle activity program for inclusion in a restorative home care service: a pilot study. Activ Adapt Aging. 2014;38(2):79-93.

44. Whitehead PJ, Drummond AER, Parry RH, Walker MF. Content and acceptability of an occupational therapy intervention in homecare re-ablement services (OTHERS). Br J Occup Ther. 2018;81 (9):535-542. doi:10.1177/0308022618766844

45. Baker DI, Gottschalk M, Eng C, Weber S, Tinetti ME. The design and implementation of a restorative care model for home care. Gerontologist. 2001;41(2):257-263. doi:10.1093/geront/41.2.257

46. Burton E, Lewin G, Boldy D. Physical activity levels of older adults receiving a home care service.. J Aging Phys Act. 2013;21 (2):140-154. doi:10.1123/japa.21.2.140

47. Tuntland H, Kjeken I, Folkestad B, Forland O, Langeland E. Everyday occupations prioritised by older adults participating in reablement. A cross-sectional study. Scand J Occup Ther. 2019;1-11.

48. Tuntland H, Kjeken I, Langeland E, et al. Predictors of outcomes following reablement in community-dwelling older adults. Clin Interv Aging. 2017;12:55-63. doi:10.2147/CIA.S125762

49. Zingmark M, Evertsson B, Haak M. Characteristics of occupational therapy and physiotherapy within the context of reablement in Swedish municipalities: A national survey. Health Soc Care Community. 2020;28(3):1010-1019. doi:10.1111/hsc.12934

50. Birkeland A, Tuntland H, Førland O, Jakobsen FF, Langeland E. Interdisciplinary collaboration in reablement-a qualitative study. J Multidiscip Healthc. 2017;10:195-203. doi:10.2147/JMDH.S133417

51. Bodker MN, Langstrup H, Christensen U. What constitutes 'good care' and 'good carers'? The normative implications of introducing reablement in Danish home care. Health Soc Care Community. 2019;27(5):e871-e878.

52. Bødker MN. Potentiality made workable-exploring logics of care in reablement for older people. Ageing Soc. 2018;39(9):2018-2041. doi:10.1017/S0144686X18000417

53. Eliassen M, Henriksen N, Moe S. The practice of support personnel, supervised by physiotherapists, in Norwegian reablement services. Physiother Res Int. 2018;24:e1754. doi:10.1002/pri.1754

54. Eliassen M, Henriksen NO, Moe S. Variations in physiotherapy practices across reablement settings. Physiother Theory Pract. 2018:1-14.

55. Eliassen M, Henriksen NO, Moe S. Physiotherapy supervision of home trainers in interprofessional reablement teams. J Interprof Care. 2018;33(5):512-518. doi:10.1080/13561820.2018.1541877

56. Gustafsson L, Anbäcken E, Elfström M, Zander V, Östlund G. Working with short-term goal-directed reablement with older adults: strengthened by a collaborative approach. Nordic J Nurs Res. 2019;2057158519850974.

57. Hansen AM, Kamp A. From carers to trainers: professional identity and body work in rehabilitative eldercare. Gend Work Organ. 2016;25(1).

58. Hjelle KM, Skutle O, Alvsvåg H, Førland O. Reablement teams' roles: a qualitative study of interdisciplinary teams' experiences. J Multidiscip Healthc. 2018;11:305-316. doi:10.2147/JMDH.S160480

59. Hjelle KM, Skutle O, Førland O, Alvsvåg H. The reablement team's voice: a qualitative study of how an integrated multidisciplinary team experiences participation in reablement. $J$ Multidiscip Healthc. 2016;9:575-585. doi:10.2147/JMDH.S115588

60. Jakobsen FA, Vik K. Health professionals' perspectives of next of kin in the context of reablement. Disabil Rehabil. 2018;41 (16):1882-1889. doi:10.1080/09638288.2018.1450452

61. Jokstad K, Skovdahl K, Landmark BT, Haukelien H. Ideal and reality; Community healthcare professionals' experiences of userinvolvement in reablement. Health Soc Care Community. 2019;27 (4):907-916. doi:10.1111/hsc. 12708
62. Meldgaard Hansen A. Rehabilitative bodywork: cleaning up the dirty work of homecare. Sociol Health Illn. 2016;38(7):1092-1105. doi:10.1111/1467-9566.12435

63. Moe A, Brataas H. Interdisciplinary collaboration experiences in creating an everyday rehabilitation model: a pilot study. J Multidiscip Healthc. 2016;9:173. doi:10.2147/JMDH.S103696

64. Moe C, Brinchmann BS. Tailoring reablement: A grounded theory study of establishing reablement in a community setting in Norway. Health Soc Care Community. 2018;26(1):113-121. doi:10.1111/hsc.12471

65. Liaaen J, Vik K. Becoming an enabler of everyday activity: health professionals in home care services experiences of working with reablement. Int $J$ Older People Nurs. 2019;14(4):e12270. doi:10.1111/opn.12270

66. Eliassen M, Lahelle A. Enhancing functional improvement in reablement - a qualitative study. Eur J Physiother. 2020;1-7. doi:10.1080/ 21679169.2020.1761449

67. Bodker MN, Christensen U, Langstrup H. Home care as reablement or enabling arrangements? An exploration of the precarious dependencies in living with functional decline. Sociol Health Illn. 2019.

68. Gustafsson LK, Ostlund G, Zander V, Elfstrom ML, Anbacken EM. 'Best fit' caring skills of an interprofessional team in short-term goaldirected reablement: older adults' perceptions. Scand J Caring Sci. 2019;33(2):498-506. doi:10.1111/scs.12650

69. Hjelle KM, Tuntland H, Førland O, Alvsvåg H. Driving forces for homebased reablement; a qualitative study of older adults' experiences. Health Soc Care Community. 2017;25(5):1581-1589. doi:10.1111/hsc.12324

70. Moe A, Ingstad K, Brataas HV. Patient influence in home-based reablement for older persons: qualitative research. BMC Health Serv Res. 2017;17(1):736. doi:10.1186/s12913-017-2715-0

71. Moe CF, Brinchmann BS. Optimising capacity-a service user and caregiver perspective on reablement. Grounded Theory Rev. 2016;15 (2): $25-40$.

72. Wilde A, Glendinning C. 'If they're helping me then how can I be independent?' The perceptions and experience of users of home-care re-ablement services. Health Soc Care Community. 2012;20 (6):583-590. doi:10.1111/j.1365-2524.2012.01072.x

73. Magne TA, Vik K. Promoting participation in daily activities through reablement: a qualitative study. Rehabil Res Pract. 2020;2020: 6506025.

74. Hjelle KM, Alvsvåg H, Førland O. The relatives' voice: how do relatives experience participation in reablement? A qualitative study. J Multidiscip Healthc. 2017;10:1-11. doi:10.2147/JMDH. $\mathrm{S} 122385$

75. Jakobsen FA, Vik K, Ytterhus B. The art of maintaining everyday life: collaboration among older parents, their adult children, and health care professionals in reablement. $J$ Multidiscip Healthc. 2019;12:269-280. doi:10.2147/JMDH.S195833

76. Moher D, Liberati A, Tetzlaff J, Altman DG. The PRISMA Group (2009). Preferred Reporting Items for Systematic Reviews and MetaAnalyses: The PRISMA Statement. PLoS Med. 6(7):e1000097. doi: doi:10.1371/journal.pmed1000097

77. Tuntland H, Espehaug B, Forland O, Hole AD, Kjerstad E, Kjeken I. Reablement in community-dwelling adults: study protocol for a randomised controlled trial. BMC Geriatr. 2014;14:139. doi:10.1186/1471-2318-14-139

78. Langeland E, Langland E, Tuntland H, et al. Study protocol for a multicenter investigation of reablement in Norway. BMC Geriatr. 2015;15(15):111. doi:10.1186/s12877-015-0108-y

79. World Health Organization. Global Strategy on Diet, Physical Activity and Health [Internet]. Geneva: World Health Organization; 2018 July. Available from: http://www.who.int/dietphysicalactivity/ factsheet_olderadults/en/. Accessed September 30, 2020.

80. Devereux-Fitzgerald A, Powell R, Dewhurst A, French DP. The acceptability of physical activity interventions to older adults: A systematic review and meta-synthesis. Soc Sci Med. 2016;158:14-23. doi:10.1016/j.socscimed.2016.04.006 


\section{Publish your work in this journal}

The Journal of Multidisciplinary Healthcare is an international, peerreviewed open-access journal that aims to represent and publish research in healthcare areas delivered by practitioners of different disciplines. This includes studies and reviews conducted by multidisciplinary teams as well as research which evaluates the results or conduct of such teams or healthcare processes in general. The journal covers a very wide range of areas and welcomes submissions from practitioners at all levels, from all over the world. The manuscript management system is completely online and includes a very quick and fair peer-review system. Visit http://www.dovepress.com/testimonials. php to read real quotes from published authors. 